\title{
hsa_circ_0072389, hsa_circ_0072386, hsa_circ_0008621, hsa_circ_0072387, and hsa_circ_0072391 aggravate glioma via miR-338-5p/IKBIP
}

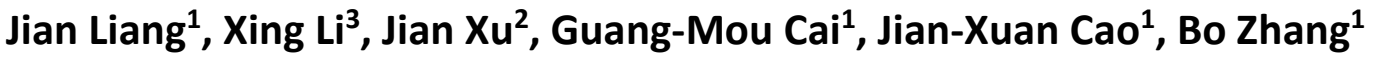 \\ ${ }^{1}$ The Second Clinical Medical College of Jinan University, Shenzhen People's Hospital, Shenzhen, China \\ 2Department of Hematology, The Second Clinical Medical College of Jinan University, Shenzhen People's Hospital, \\ Shenzhen, China \\ ${ }^{3}$ School of Medicine, Southern University of Science and Technology, Shenzhen, China
}

Correspondence to: Bo Zhang; email: zhang.bo@szhospital.com

Keywords: miR-338-5p, circRNA, IKBIP, ceRNA, glioma

Received: February 24, $2021 \quad$ Accepted: November 11, 2021

Published: December 12, 2021

Copyright: () 2021 Liang et al. This is an open access article distributed under the terms of the Creative Commons Attribution License (CC BY 3.0), which permits unrestricted use, distribution, and reproduction in any medium, provided the original author and source are credited.

\begin{abstract}
Glioma is a primary intracranial tumor with high morbidity and mortality. We acquired miR-338-5p, which suppresses the development of glioma, from the GEO and CGGA databases. In addition, we predicted that hsa_circ_0072389, hsa_circ_0072386, hsa_circ_0008621, hsa_circ_0072387, and hsa_circ_0072391 could relieve the silencing of IKBIP by miR-338-5p. By analyzing genes related to IKBIP expression, possible pathways affecting glioma were identified. This study provides new ideas for investigating multiple circRNAs in ceRNAs.
\end{abstract}

\section{INTRODUCTION}

Glioma is the most common primary malignant brain tumor and accounts for approximately half of all intracranial primary tumors [1]. The current effective treatment methods are surgery, radiotherapy, and chemotherapy, the survival of patients posttreatment is still suboptimal [2]. Fortunately, the development of molecular biotechnology provides new ideas for the diagnosis and treatment of glioma.

Circular RNA is a type of noncoding RNA lacking both a $5^{\prime}$ end cap and a $3^{\prime}$ end polytail that induces a circular RNA structure via covalent bonds [3]. Due to their closed circular structure, circRNAs are seldom degraded by exonuclease, which makes them more stable than linear RNAs [4]. An increasing number of studies show that circRNAs participate in the occurrence and development of a variety of cancers [5]. By regulating gene transcription, interacting with proteins, or translating into polypeptides, circRNAs can participate in the regulation of cancer $[6,7]$. However, current research mainly regards circRNA as a competing endogenous RNA [8]. As miRNAs can not only silence downstream genes by targeting mRNAs but also bind sites located in circRNAs (miRNA response elements, MREs), circRNAs can prevent the suppression of target genes by competitively binding to miRNAs [9]. For example, Cen $\mathbf{J}$ et al. discovered that circular RNA circSDHC sponges adsorb miR-127-3p, which upregulates the expression of $\mathrm{CDKN} 3$ and $\mathrm{E} 2 \mathrm{~F} 1$ and promotes the proliferation and metastasis of renal cell carcinoma [10]. Chen LY et al. revealed that the circular RNA circ-ERBIN promoted colorectal cancer by sponging miR-125a-5p and miR-138-5p, thereby alleviating the silencing of 4EBP-1 [11]. According to multiple studies, we found that a miRNA could be sponged by multiple circRNAs in different cancers. For example, miR-942-5p could be adsorbed by hsa_circ_0015756 in ovarian cancer, circRNA-AKT1 in cervical cancer, and circ-CEP85L in gastric cancer [12-14]. However, 
studies of the mechanism by which multiple circRNAs regulate miRNAs in the same cancer have not been performed.

In this study, we found that miR-338-5p is differentially expressed between glioma tumor and normal tissues in GSE datasets (GSE139031, GSE25632, GSE103228, GSE65626), indicating that miR-338-5p may have a stable effect on the occurrence or development of glioma. By analyzing the data in several databases, including circBANK, GEO, and circinteractome, we deduced that hsa_circ_0072389, hsa_circ_0072386, hsa_circ_0008621, hsa_circ_0072387, and hsa_circ_0072391 may aggravate glioma by combining with miR-338-5p. In addition, with the circPrimer and circBase databases, we found that these 5 circRNAs all originated from HMGCS1. Moreover, we found that IKBIP may be the target gene of miR-338-5p by analyzing multiple databases, which involved miRwalk, miRDB, TargetScan, GEO, and GEPIA. Therefore, we believe that hsa_circ_0072389, hsa_circ_0072386, hsa_circ_0008621, hsa_circ_0072387, and hsa_circ_0072391 promote the expression of IKBIP by binding with miR-338-5p. Then, we verified the relationship among circRNAs (hsa_circ_0072389, hsa_circ_0072386, hsa_circ_0008621, hsa_circ_0072387, hsa_circ_0072391), miR-338-5p and IKBIP by Western blot. With the Pathcards and GEPIA databases, we inferred that IKBIP may promote the development of glioma NF- $\kappa$ B, JAK/STAT and TGF $\beta / S M A D$ signaling pathways. In summary, we believe that hsa_circ_0072389, hsa_circ_0072386, hsa_circ_0008621, hsa_circ_0072387, and hsa_circ_0072391 induce the NF- $\kappa$ B and JAK/STAT pathways to aggravate glioma via miR-338-5p/IKBIP.

\section{MATERIALS AND METHODS}

\section{Public database collection}

High-throughput data on circRNAs, miRNAs and mRNAs in glioma were obtained from the GEO database. The screening standard for circRNA was $|\log \mathrm{FC}| \geq 1.5 p<0.05$ and $|\log \mathrm{FC}| \geq 2 p<0.05$ for miRNA and mRNA. The whole screening process was implemented with the limma R package [15].

\section{Prediction of circRNAs and mRNAs}

The prediction of circRNAs binding with miR-338-5p was based on the circBANK database (http://www.circbank.cn/), and mRNAs that targeted miR-338-5p were discovered according to the results from the miRwalk (http://mirwalk.umm.uniheidelberg.de/), miRDB (http://mirdb.org/), and targetscan databases (http://www.targetscan.org/vert 72/). All these results indicated the presence of conserved 8-mer and 7-mer sites that match the seed region of miR-338-5p [16, 17].

\section{Prediction of proteins bind to circRNA}

The circinteractome database (https://circinteractome.nia.nih.gov/) was employed to reveal proteins that interact with circRNA. In addition, the TargetScan prediction tool enables the prediction and binding sites for RBPs on reported circRNAs that have yet to be mapped [18].

\section{KEGG and GO Enrichment Analysis}

KEGG was used to analyze the pathways involved in mRNAs, and GO was used to analyze molecular function (MF), biological process (BP), and cellular component. All operations were implemented by using the Clusterprofiler R package [19].

\section{PPI (Protein-protein interaction)}

The protein-protein interaction (PPI) network was constructed by the STRING v11 database (https://stringdb.org/) [20].

\section{circRNA secondary structure and MFE structure}

The sequences of circRNAs were obtained from circbase (http://circrna.org/) and further entered into the RNAfold database (http://rna.tbi.univie.ac.at/) to acquire the secondary structures and MFE structures of circRNAs.

\section{Analysis of survival rate}

The overall survival time and gene expression of glioma patients were obtained from the GEPIA database (http://gepia.cancer-pku.cn/). Kaplan-Meier plots were used to analyze the relationship between the survival time and gene expression of glioma patients. The hazard ratio [21] and 95\% confidence intervals were acquired by the statistical software SPSS 19.0.

\section{The correlation of gene expression}

Pathway-related genes were obtained from the Pathcards database (https://pathcards.genecards.org/), and the correlation between IKBIP and gene expression in glioma was obtained through the GEPIA database.

\section{Cell culture}

The glioma cell Line U251 was cultured in MEM containing $10 \%$ fetal bovine serum and $1 \%$ glutamine, 
and the cell culture environment was $5 \%$ carbon dioxide in a $37^{\circ} \mathrm{C}$ incubator.

\section{RNA extraction, $\mathbf{R T}-\mathbf{q P C R}$}

RNA was extracted by TRIzol reagent, and RT-qPCR was performed by a 7500 real-time PCR system (Thermo Fisher Scientific). The miR-338-5p inhibitor was obtained from Boshang Biotechnology (Shanghai). Taking GAPDH as a reference, the primer sequences were as follows:

$\begin{array}{ll}\text { hsa_circ_0072389: } & \begin{array}{l}\text { Left primer: CAGGTGGAGTTGGAGCAGTA } \\ \text { Right primer: CAGCGGTCTAATGCACTGAG }\end{array} \\ \text { hsa_circ_0072386: } & \begin{array}{l}\text { Left primer: GCTGCTGTCTTCAATGCTGT } \\ \text { Right primer: TTTGGCCCAATTAGCAGAGC }\end{array} \\ \text { hsa_circ_0008621: } & \text { Left primer: GCTGCCACTCTGTACTCTCT } \\ & \text { Right primer: GGTGTCCTCTCTGAGCTTCA } \\ \text { hsa_circ_0072387: } & \text { Left primer: AGACCGCTGCTATTCTGTCT } \\ & \text { Right primer: TCATTCAGCAACATCCGAGC } \\ \text { hsa_circ_0072391: } & \text { Left primer: AGACCGCTGCTATTCTGTCT } \\ & \text { Right primer: TCATTCAGCAACATCCGAGC } \\ \text { GAPDH: } & \text { Forward: 5'-GGTCGGAGTCAACGGATTTG-3' } \\ & \text { Reverse: 5'-ATGAGCCCCAGCCTTCTCCAT-3' }\end{array}$

\section{Western blot}

A BCA Protein Detection Kit (Thermo Scientific ${ }^{\mathrm{TM}}$, Shanghai, China) was used to detect the protein concentration. SDS $(5 \times)$ was added to the total protein, and the mixture was further heated at $100^{\circ} \mathrm{C}$ for 10 minutes. The protein was isolated by SDS-PAGE and transferred to polyvinylidene fluoride (PVDF) film. Five percent skim milk was sealed at room temperature for 2 hours and further incubated with primary antibody in a shaking bottle at $4{ }^{\circ} \mathrm{C}$ for $8-12$ hours. Then, we washed the film with Tris-buffered saline and Tween 20 (TBST) 3 times, and each wash lasted 10 minutes. After that, the secondary antibody was incubated with film at room temperature for 1 hour and washed with TBST once more 3 times (10 min each time). Proteins were observed by enhanced chemiluminescence.

\section{Statistical analyses}

Student's $t$-test was used to determine statistically significant differences. When $P<0.05$, the difference was statistically significant. The statistical analysis software used in this study was SPSS 19.0.

\section{Data availability statement}

The datasets used in the project are available from the corresponding author. The data that support the findings of this study are openly available in GEO at https://www.ncbi.nlm.nih.gov/geo/.

\section{RESULTS}

\section{miR-338-5p inhibited the progression of glioma}

A number of studies have shown that miRNAs can promote or prevent the occurrence and development of glioma. In this study, we selected GEO datasets (GSE139031, GSE25632, GSE103228, and GSE65626) with large sample sizes for analysis. When comparing gene expression in tumor tissues with gene expression in normal tissues, there were 3556 differential genes in GSE139031, 1145 differential genes in GSE25632, 6658 differential genes in GSE103228, and 3556 differential genes in GSE65626 (Figure 1A-1D). After screening $(|\operatorname{logFC}| \geq 2, p<0.05)$, we found 1426 differential genes in GSE139031 (1312 upregulated genes and 114 downregulated genes), 32 differential genes in GSE25632 (21 upregulated genes and 11 downregulated genes), 26 differential genes in GSE103228 (4 upregulated genes and 22 downregulated genes), and 30 differential genes in GSE65626 (20 upregulated genes and 10 downregulated genes) (Figure $1 \mathrm{E}-1 \mathrm{H})$. To identify miRNAs stably expressed in glioma, we intersected these differential genes in four datasets and found that miR-338-5p was the only intersection of the four datasets (Figure 1I). By analyzing the CGGA database data, we found that patients with glioma in the miR-338-5p high expression group had a longer survival time, and the expression of miR-338-5p in patients with WHO2 was significantly higher than those with WHO3-4 (Figure $1 \mathrm{~J}-1 \mathrm{~K}$ ); therefore, we speculate that miR-338-5p can inhibit the progression of glioma.

hsa_circ_0072389, hsa_circ_0072386, hsa_circ_0008621, hsa_circ_0072387, and hsa_circ_0072391 bind to miR$338-5 p$ in glioma

circRNA can target miRNA to prevent the degradation of mRNA induced by miRNA [22]. To explore circRNAs binding to miR-338-5p, 4872 circRNAs were screened out by using circBANK. In addition, we revealed that 472 circRNAs in GSE146463 $(|\log F C| \geq$ $1.5, p<0.05)$ were differentially expressed between glioma tissues and normal tissues, which included 351 upregulated circRNAs and 121 downregulated circRNAs (Figure 2A and 2B). We found that hsa_circ_0072389, hsa_circ_0072386, hsa_circ_0008621, hsa_circ_0072387, hsa_circ_0072391, hsa_circ_0044234, and hsa_circ_0001685 were not only differentially expressed in GSE146463 but also had separate binding sites for miR-338-5p (Figure 2C). In addition, binding 
sites for miR-338-5p located in these 7 circRNAs were analyzed in the CircInteractome database (Supplementary Figure $1 \mathrm{~A}-1 \mathrm{G})$. AGO2 is the core component of RISC [21], which connects target sites of miRNA and mRNA [23]. The formation of a ternary complex of circRNA-miRNA-AGO2 can also prove that circRNA interacts with miRNA [24]. We predicted the proteins that may bind to hsa_circ_0072389, hsa_circ_0072386, hsa_circ_0008621, hsa_circ_0072387, hsa_circ_0072391, hsa_circ_0044234, and hsa_circ_0001685 by searching the CircInteractome database and obtained the number
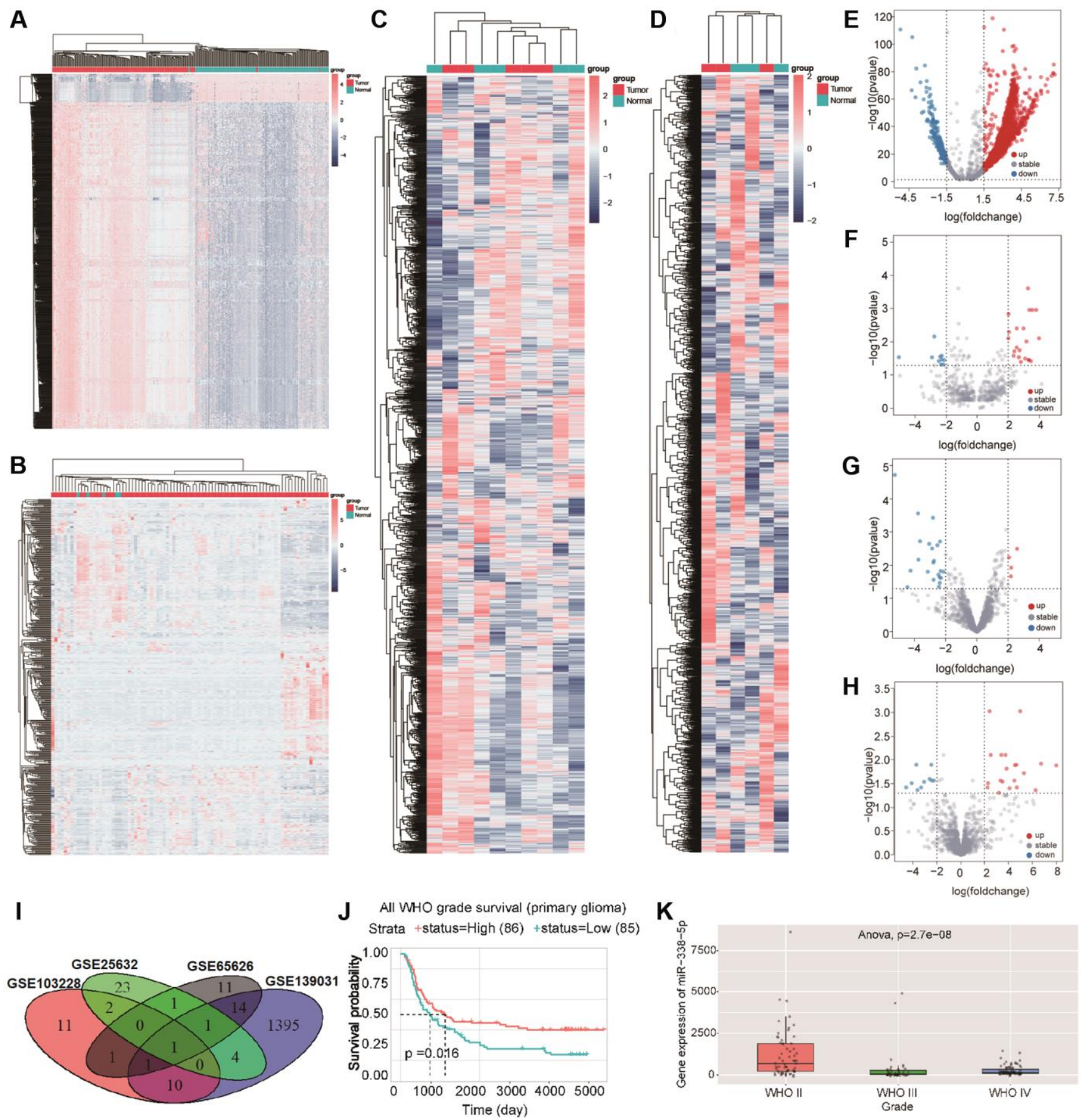

Figure 1. (A-D) Cluster analysis showed the differential genes in GSE139031, GSE25632, GSE103228, and GSE65626 in turn .red dots indicated upregulated differential genes and blue dots indicated downregulated differential genes. (E-H) volcano map showed the differential genes in GSE139031, GSE25632, GSE103228, and GSE65626, Red dots represent differential genes with logFC $\geq 2, p<0.05$, blue dots represent differential genes with $\operatorname{logFC} \leq 2, p<0.05$. (I)The Venn diagram shows the intersection of differential genes between GSE139031, GSE25632, GSE103228, and GSE65626. (J) Survival analysis demonstrated the effect of miR-338-5p on glioma. (K) The box diagram showed that the expression level of miR-338-5p in WHO II was significantly higher than that in WHO III and WHO IV. 
of binding sites with AGO2 (Figure 2D-2J). To screen for possible circRNAs binding to miR-338-5p, we selected hsa_circ_0072389, hsa_circ_0072386, hsa_circ_0008621, hsa_circ_0072387, and hsa_circ_0072391, which had great than 5 binding sites with $\mathrm{AGO} 2$, for further research.
Composition, secondary structure and sequence information of circRNAs

Most circRNAs are composed of exons, followed by introns and exons, and few are composed of introns [25]. We obtained the composition and sequence of
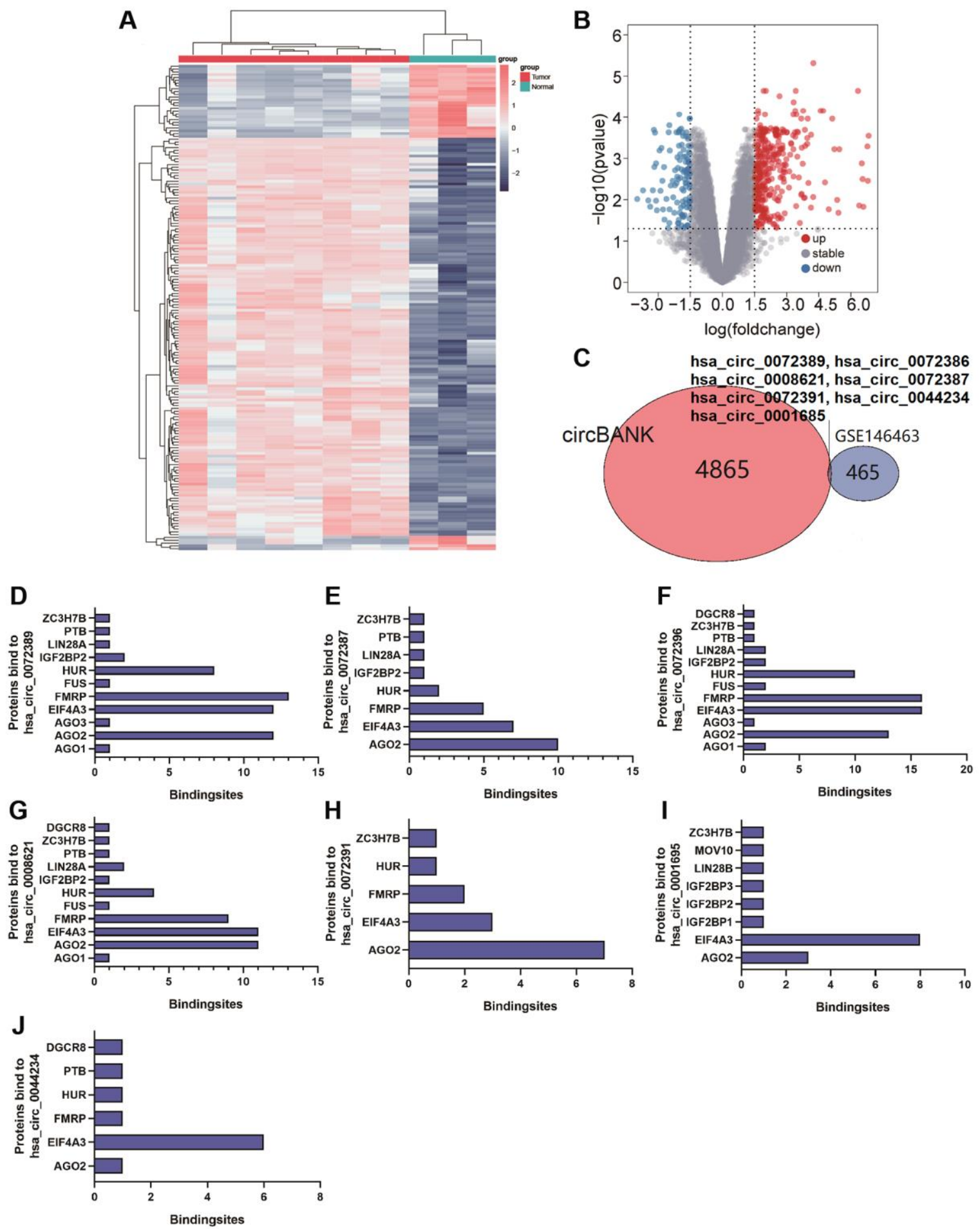

Figure 2. (A and B) Cluster analysis and volcano map demonstrate differential circRNAs in gliomas of GSE146463. (C) Venn Diagram shows circRNAs bound to miR-338-5p. (D-J) The bar graph shows proteins and the number of binding sites bound to hsa_circ_0072389, hsa_circ_0072386, hsa_circ_0008621, hsa_circ_0072387, hsa_circ_0072391, hsa_circ_0044234, hsa_circ_0001685. 
Table 1. CircRNAs binding to miR-338-5p.

\begin{tabular}{|c|c|c|c|}
\hline circBASEname & Gene symbol & Location & Sequence \\
\hline hsa_circ_0072389 & HMGCS1 & chr5:43294157-43299077 & $\begin{array}{l}\text { TACTCTCTTAAAGTCACACAAGATGCTACA } \\
\text { CCGGCTCTTTCACCATGCCTGGATCACTTC }\end{array}$ \\
\hline hsa_circ_0072386 & HMGCS1 & chr5:43292575-43299077 & $\begin{array}{l}\text { CTTTGGATGAAGGAGTAGGACTTGTGCATT } \\
\text { CAAACATAGCAACTGAGCTCTTTCACCATG }\end{array}$ \\
\hline hsa_circ_0008621 & HMGCS1 & chr5:43292575-43297268 & $\begin{array}{l}\text { TTCAAACATAGCAACTGAGGGCTTCGTGGG } \\
\text { ACACATATGCAACATGCCTATGATTTTTAC }\end{array}$ \\
\hline hsa_circ_0072387 & HMGCS1 & chr5:43294157-43297268 & $\begin{array}{l}\text { CAAGATGCTACACCGGGGCTTCGTGGGAC } \\
\text { ACATATGCAACATGCCTATGATTTTTACAAG }\end{array}$ \\
\hline hsa_circ_0072391 & HMGCS1 & chr5:43295853-43297268 & $\begin{array}{l}\text { GAGATAAAAATAGTATCTATAGTGGCCTGG } \\
\text { AAGCCTTTGGGGCTTCGTGGGACACATATG }\end{array}$ \\
\hline hsa_circ_0044234 & $\mathrm{CDC} 27$ & chr17:45247282-45249430 & $\begin{array}{l}\text { CATTGTTGGGACATGTATATTGTACACTCA } \\
\text { GAAGAAGCCTTGTTTTTACTGGCAACCTGT }\end{array}$ \\
\hline hsa_circ_0001685 & MPP6 & chr7:24663284-24690331 & $\begin{array}{l}\text { ACCAAGTTATAGAGATACCATTACTCCTCA } \\
\text { ACAGCAATGCAGCAAGTCTTGGAAAACCTT }\end{array}$ \\
\hline
\end{tabular}

hsa_circ_0072389,

hsa_circ_0008621,

hsa_circ_0072386,

hsa_circ_0072387, and

hsa_circ_0072391 from the circPrimer and circBase databases (Supplementary Figure 1H-1L, Supplementary Figure 2). In addition, the secondary structure and minimum free energy (MFE) structure of circRNAs were acquired from the RNAfold database (Supplementary Figure 3A-3E). Interestingly, we found that hsa_circ_0072389, hsa_circ_0072386, hsa_circ_0008621, hsa_circ_0072387, and hsa_circ_0072391 were all transcribed by the same host gene HMGCS1 on chromosome 5, and they all contained EXON4 and EXON5 (Table 1, Supplementary Figure $1 \mathrm{H}-1 \mathrm{~L})$. In addition, 5 circRNAs were composed of single-stranded structures, stems, hairpin loops, convex loops, inner loops, and multibranched loops, which provide directions for further exploration of the genes regulating transcription, interaction with proteins, or translation into polypeptides.

\section{IKBIP binds to miR-338-5p and aggravates glioma}

We further predicted mRNAs that might bind miR-3385p. In GSE4290, we found 1319 differentially expressed genes $(|\log \mathrm{FC}| \geq 2, p<0.05)$, which involved 451 upregulated genes and 868 downregulated genes (Figure 3A and 3B). In addition, we obtained 879, 1328, and 4812 mRNAs that may bind to miR-338-5p from the miRwalk, miRDB, and TargetScan databases, respectively. By comparing differential mRNAs in the GSE4290, miRwalk, miRDB, and TargetScan databases, we found that 100 differential mRNAs may not only be correlated with glioma but also bind to miR338-5p (Figure 3C). Further analysis of these 100 genes by KEGG and GO revealed that these 100 genes tend to regulate signal transduction in pathways, biological processes, cell components, and molecular functions
(Supplementary Figure 4A-4D). According to the results described above, we plotted the circRNAmiRNA-mRNA network (Supplementary Figure 5). In addition, using the STRING database, we analyzed the interaction relationships among proteins originating from 100 mRNAs (Supplementary Figure 6). Furthermore, to screen glioma-related mRNAs, we analyzed the effects of these 100 mRNAs on the prognosis of glioma patients in the GEPIA database. We found that patients with low expression of CCBE1, IKBIP, NRG1, and RGS4 had longer survival times (Figure 3D-3G). In addition, we separately acquired the binding sites of CCBE1, IKBIP, NRG1, and RGS4 with miR-338-5p from the TargetScan database (Supplementary Figure 4E-4H). In the GEPIA database, we found that IKBIP was upregulated in glioma relative to normal tissue, while CCBE1, NRG1, and RGS4 were downregulated in glioma (Figure $3 \mathrm{H}$ ). Since we found that patients with low expression of CCBE1, IKBIP, NRG1, and RGS4 had a better prognosis than patients with high expression, there was a contradiction between the expression of CCBE1, NRG1, and RGS4 and their effect on prognosis. Therefore, we hypothesized that IKBIP was regulated by miR-338-5p in glioma. In summary, we constructed the hsa_circ_0072389, hsa_circ_0072386, hsa_circ_0008621, hsa_circ_0072387, hsa_circ_0072391, miR-338-5p, and IKBIP networks (Figure 3I).

hsa_circ_0072389, hsa_circ_0072386, hsa_circ_0008621, hsa_circ_0072387, and hsa_circ_0072391 promote the expression of IKBIA, while miR-338-5p has the opposite effect

To verify the relationship among hsa_circ_0072389, hsa_circ_0072386, hsa_circ_0008621, hsa_circ_0072387, and hsa_circ_0072391 with IKBIP, 
we knocked down hsa_circ_0072389, hsa_circ_0072386, hsa_circ_0008621, hsa_circ_0072387, and hsa_circ_0072391 in U251 glioma cells separately (Figure 4A-4E). Then, Western blotting was used to determine the expression of IKBIP in U251 glioma cells and found that the expression of
IKBIP protein was downregulated relative to that in the normal control group (Figure 4G). In addition, we inhibited the expression of miR-338-5p with a miR$338-5 p$ inhibitor (Figure 4F) and revealed that the expression of IKBIP was upregulated relative to that in the normal control group.
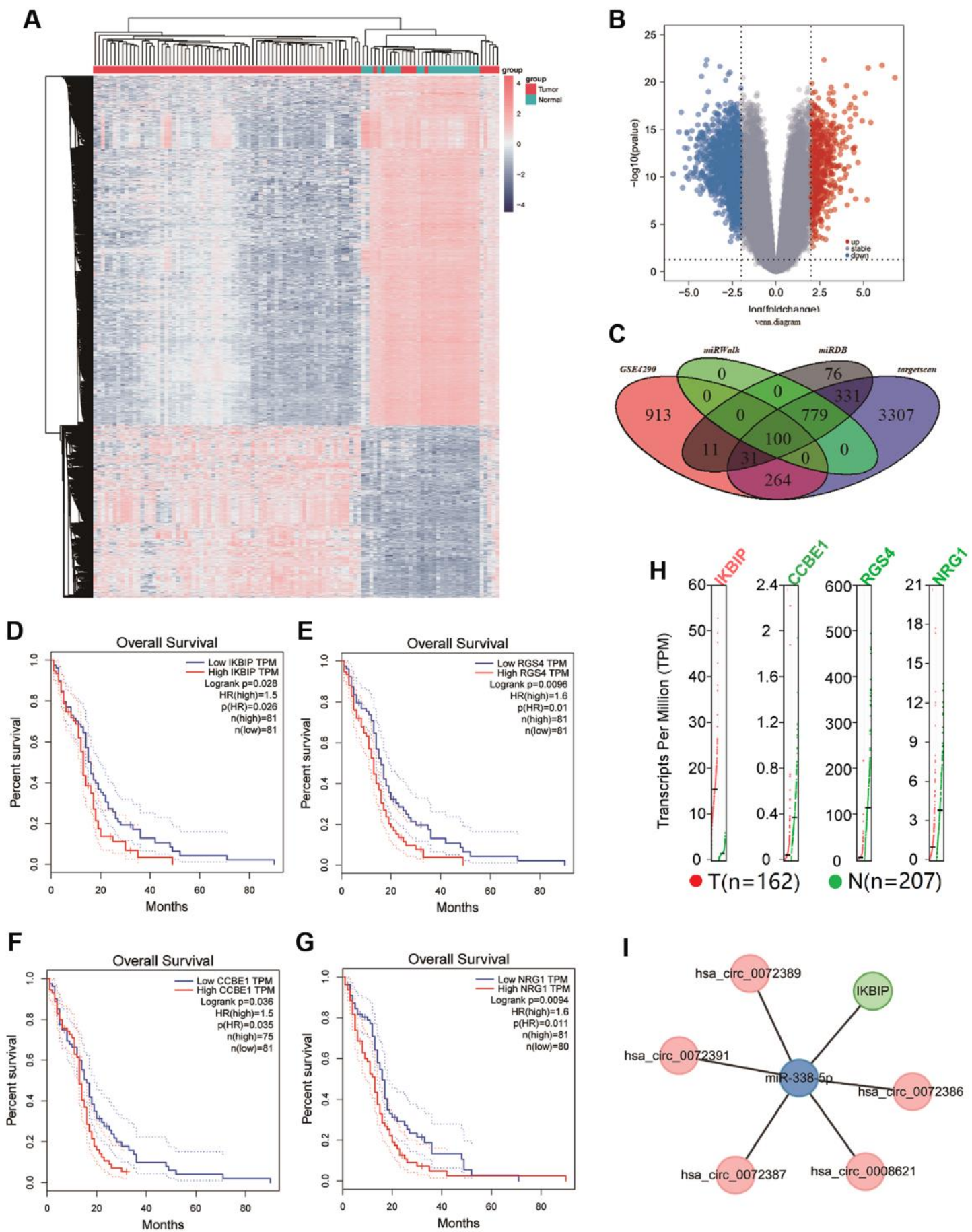

Figure 3. (A and B) Cluster analysis and volcano map demonstrate differential mRNAs in gliomas of GSE4290. (C) The Venn diagram shows the intersection of differential genes between GSE4290, miRwalk database, miRDB database, and targetscan database. (D-G) Survival curve shown the correlation between the expression of CCBE1, IKBIP, NRG1, and RGS4 with overall survival time $(p<0.05)$. (H) Gene Expression Profile of IKBIP, CCBE1, NRG1, and RGS4, red dots indicated the expression of tumor tissue, green dots indicated the expression of normal tissue. (I) Network of hsa_circ_0072389, hsa_circ_0072386, hsa_circ_0008621, hsa_circ_0072387, hsa_circ_0072391, miR-338-5p, and IKBIP. 
Pathways that may be affected by IKBIP in glioma

Because genes play different roles in different cancers [26], we explored the pathways that may be affected by IKBIP in glioma by searching the pathcards and GEPIA databases. We searched for genes involved in 8 common pathways (NF- $\kappa \mathrm{B}$ signaling pathway, PI3K/Akt signaling pathway, MAPK signaling pathway, JAK/STAT signaling pathway, TGF $\beta /$ SMAD signaling pathway, Wnt/ $\beta$-catenin signaling pathway, Notch signaling pathway, Hedgehog signaling pathway) in the PathCards database and queried the expression of these genes in glioma by analyzing data from the GEPIA database. Then, we found that the expression of the genes correlated with IKBIP (Supplementary Figures 7-14). As not all genes involved in pathways
A

$\square$ sh-hsa_circ_0072389

$\square \mathrm{NC}$

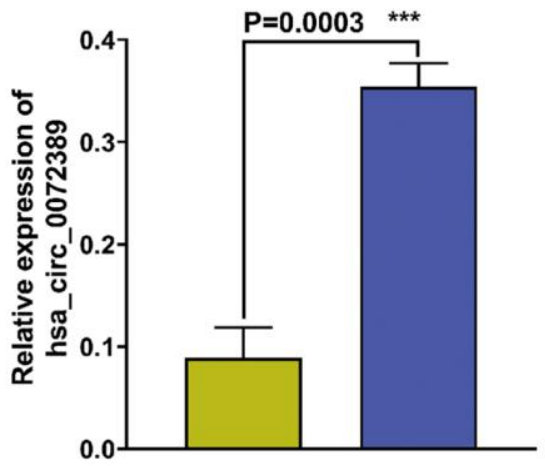

D
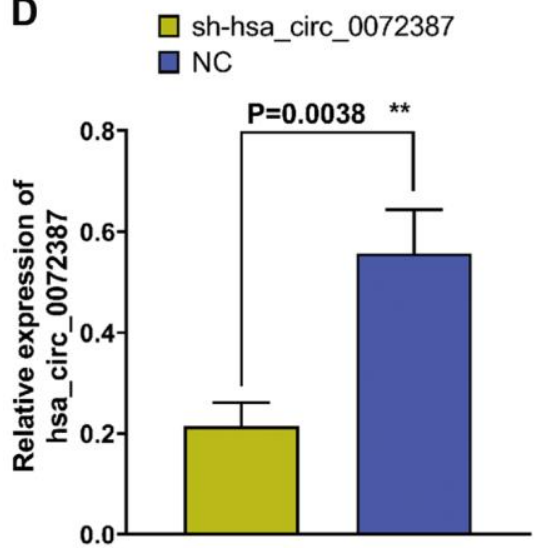

B
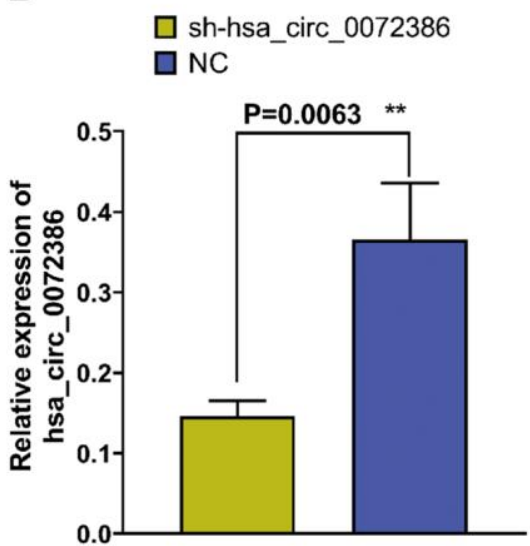

E

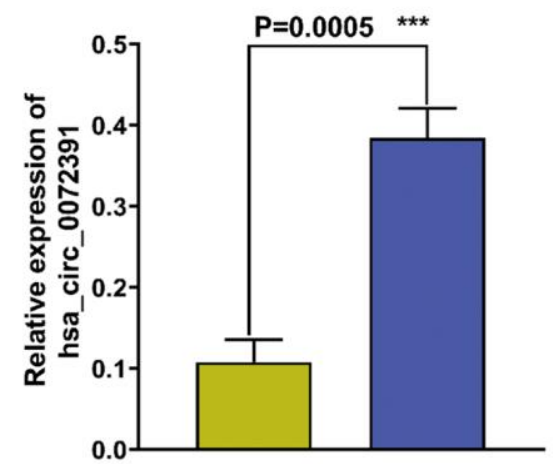

C

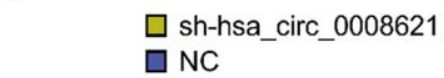

F $\square$ miR-338-5p inhibitor $\square \mathrm{NC}$

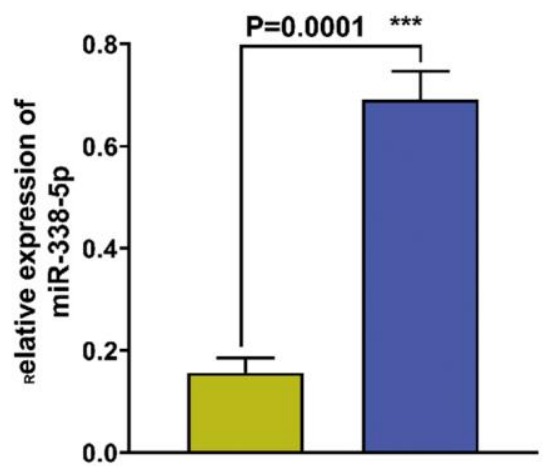

G

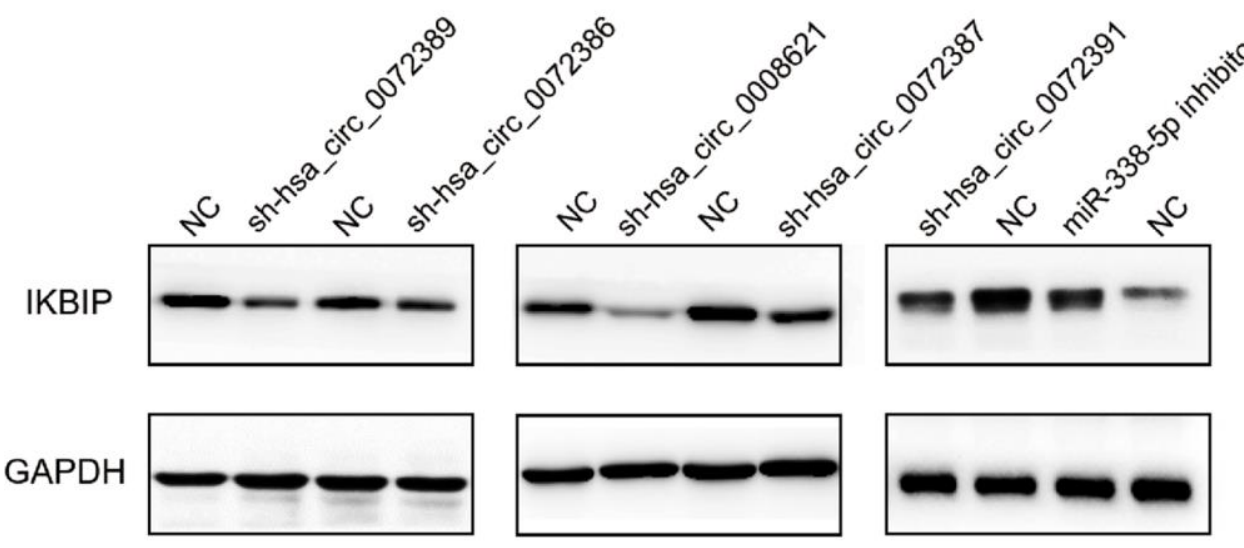

Figure 4. (A-E) RT-qPCR verified the efficiency of knocking down hsa_circ_0072389, hsa_circ_0072386, hsa_circ_0008621, hsa_circ_0072387, hsa_circ_0072391 in glioma U251, respectively. (F) RT-qPCR verified the efficiency of miR-338-5p inhibitor on miR-338$5 p$ expression inhibition. (G) Western blot shown the effect of hsa_circ_0072389, hsa_circ_0072386, hsa_circ_0008621, hsa_circ_0072387, hsa_circ_0072391, miR-338-5p on IKBIP protein expression. 
could exert an impact on glioma, we screened significant genes affecting the survival of glioma patients, which included TNFRSF1A $(p=5.9$ e-9, $\mathrm{R}=$ $0.44)$, MALT1 ( $p=9.7 \mathrm{e}-9, \mathrm{R}=0.37)$, LY96 ( $p=1 \mathrm{e}-$ $10, \mathrm{R}=0.48)$, NFKB2 ( $p=2.5 \mathrm{e}-8, \mathrm{R}=0.42), \mathrm{CD} 14(p$ $=7 \mathrm{e}-8, \mathrm{R}=0.41)$ in the NF- $\kappa \mathrm{B}$ signaling pathway, SOCS3 $(P=5$ e-8, R $=0.41)$, MAP2K3 $(p=2.4$ e-9, $\mathrm{R}$
$=0.45)$, JAK3 $(p=1.7 \mathrm{e}-5, \mathrm{R}=0.33), \operatorname{IL} 27 \mathrm{RA}(p=5.9$ $\mathrm{e}-8, \mathrm{R}=0.41)$, IL13RA1 ( $p=3.5 \mathrm{e}-13, \mathrm{R}=0.53)$ in the JAK/STAT signaling pathway, IL27RA ( $p=5.9 \mathrm{e}-8, \mathrm{R}$ $=0.41)$, and IL13RA1 $(p=3.5)$. These were not only correlated with IKBIP expression $(p<0.001,|\mathrm{R}|>0.3$ ) [27] but also affected the prognosis of glioma patients. (Figure 5, Supplementary Figure 15). Therefore, we

\section{signaling pathways}

A

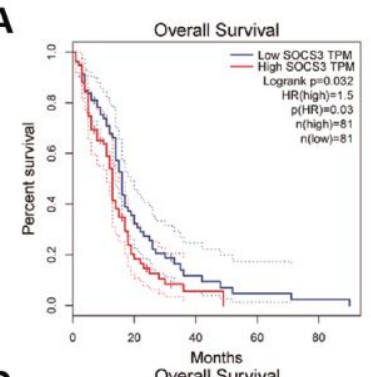

D

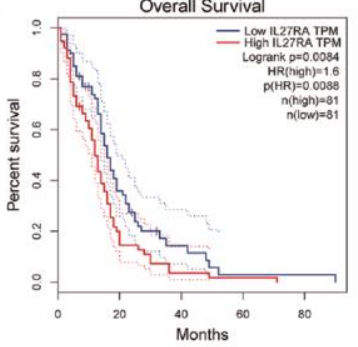

G

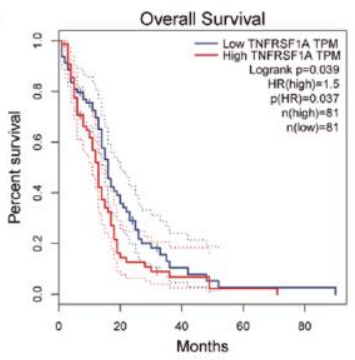

J

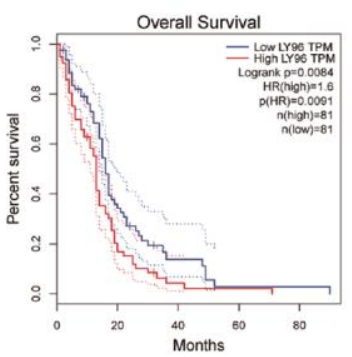

M

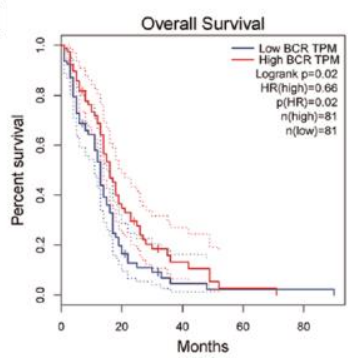

B

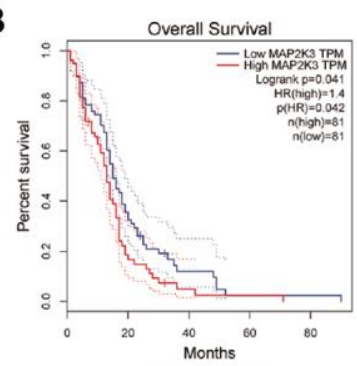

E

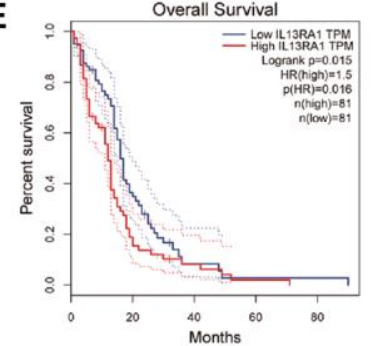

H

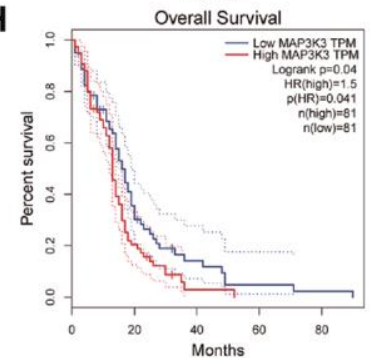

K

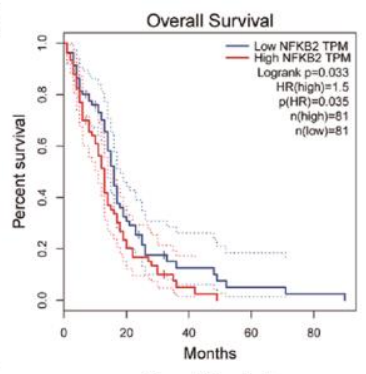

N

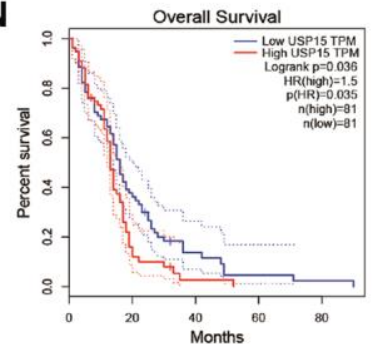

C

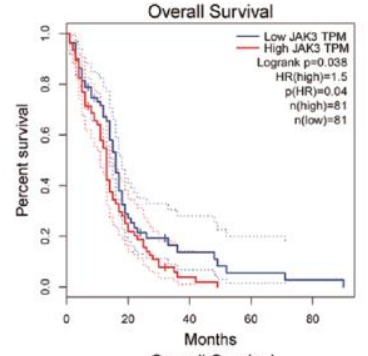

F

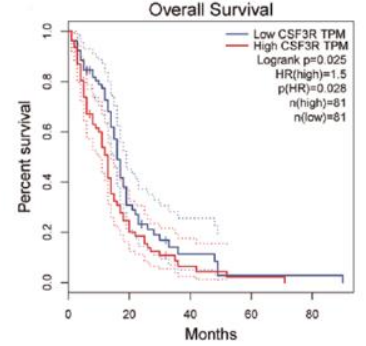

I

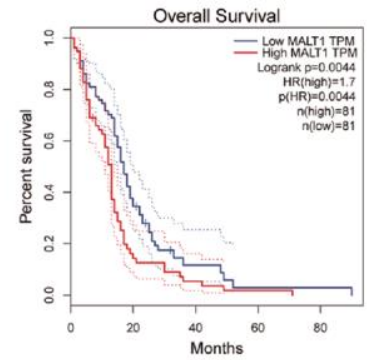

L

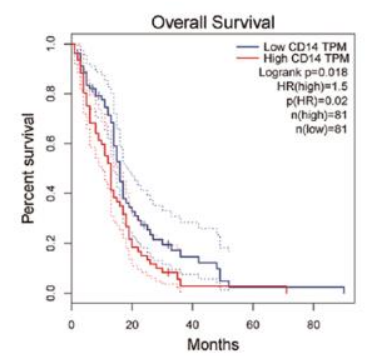

Figure 5. Survival analysis shown the effect of genes related to IKBIP in the (A-F) JAK/STAT signaling pathway, (G-M) NF-KB signaling pathway and (N) TGFB/SMAD signaling pathway to glioma patients. 
speculated that IKBIP may induce the development of glioma through the NF- $\mathrm{B}$ signaling pathway, JAK/STAT signaling pathway and TGF $\beta / S M A D$ signaling pathway.

\section{Diagram of circRNA-miRNA-mRNA pathways}

hsa_circ_0072386, hsa_circ_0008621, hsa_circ_0072387, and hsa_circ_0072391 can bind to miR-338-5p, and miR-338-5p can target IKBIP, which is coexpressed with genes in the NF- $\kappa \mathrm{B}$ signaling pathway and JAK/STAT signaling pathway. Therefore, we speculate that after being transcribed from the host gene HMGCS1, pre-RNAs are cleaved into mRNAs and circRNAs through splicing and modification. In these circRNAs, hsa_circ_0072389, hsa_circ_0072386, hsa_circ_0008621, hsa_circ_0072387, and hsa_circ_0072391 are transported between or within cells, which bind to miR-338-5p and hinder the silencing of IKBIP mRNA. Then, the upregulation of IKBIP results in the activation of the NF- $\mathrm{KB}$ signaling pathway, JAK/STAT signaling pathway and TGF $\beta / S M A D$ signaling pathway, which worsen the deterioration of glioma (Figure 6).

\section{DISCUSSION}

In GSE139031, GSE25632, GSE103228, and GSE65626, we found that miR-338-5p was expressed at low levels in glioma. In the CGGA database, we further verified that glioma patients with high miR-338-5p expression had a better prognosis. Meanwhile, a great number of studies have also shown that miR-338-5p is able to inhibit the proliferation and invasion of glioma cells [28-30]. Therefore, miR-338-5p was selected as a candidate gene for subsequent study. By searching for the presence of conserved 8-mer, 7-mer, and 6-mer sites that match the seed region of miR-338-5p, we predicted circRNAs and mRNA to bind with miR-338-5p [16]. After analyzing the GSE146463, circBANK and circInteractome databases, we found that hsa_circ_0072389, hsa_circ_0072386, hsa_circ_0008621, hsa_circ_0072387, and hsa_circ_0072391 in glioma were most likely to target miR-338-5p. Interestingly, we found that hsa_circ_0072389, hsa_circ_0072386, hsa_circ_0008621, hsa_circ_0072387, and hsa_circ_0072391 were transcribed by the same host gene HMGCS1. Moreover, by using the GEPIA,

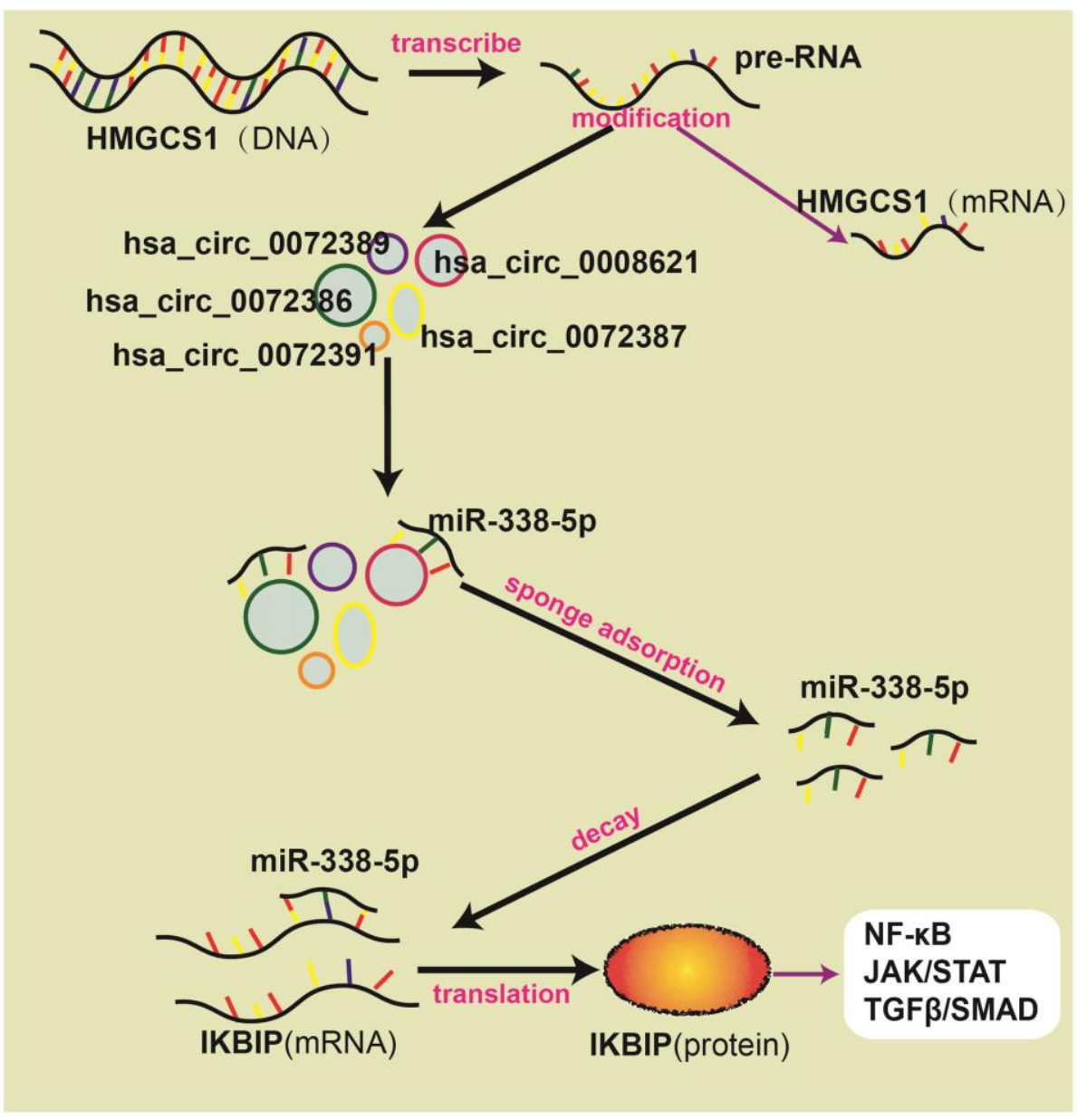

Figure 6. Schematic diagram about ceRNA network. 
miRwalk, miRDB, and TargetScan databases and GSE4290, IKBIP was found to facilitate the development of glioma and may be inhibited by miR338-5p, which induced us to establish a circRNAmiRNA-miRNA network. Furthermore, Western blot analysis verified that downregulation of hsa_circ_0072389, hsa_circ_0072386, hsa_circ_0008621, hsa_circ_0072387, and hsa_circ_0072391 decreased the expression of IKBIP, which could be reversed after inhibiting miR-338-5p. To confirm which pathways might be regulated by IKBIP, we selected the PathCards database to identify all genes in eight common cancer-related pathways (NF- $\mathrm{KB}$ signaling pathway, PI3K/Akt signaling pathway, MAPK signaling pathway, JAK/STAT signaling pathway, TGF $\beta /$ SMAD signaling pathway, Wnt/ $\beta$-catenin signaling pathway, Notch signaling pathway, Hedgehog signaling pathway). Genes associated with IKBIP expression were screened by the GEPIA database. However, although these screened genes of 8 common cancer-related pathways may be correlated with IKBIP, they may not affect glioma; therefore, we further performed survival analysis of these genes in glioma by using the GEPIA database. We found that genes in the NF- $\mathrm{BB}$, JAK/STAT and TGF $\beta / S M A D$ signaling pathways were not only correlated with IKBIP in gene expression but also affected the survival of glioma patients, which indirectly indicated that these 3 pathways (NF- $\kappa B$, JAK/STAT and TGF $\beta / S M A D$ signaling pathways) may interact with IKBIP to affect the progression of glioma. To acquire a more accurate result, the screening criteria for genes related to IKBIP in expression were stricter $(p<0.001,|\mathrm{R}|>0.3)$ [27]. Then, we found that TNFRSF1A $(p=5.9$ e-9, $\mathrm{R}=0.44)$, MALT1 $(p=9.7$ $\mathrm{e}-9, \mathrm{R}=0.37), \mathrm{LY} 96(p=1 \mathrm{e}-10, \mathrm{R}=0.48)$, NFKB2 $(p=2.5$ e- $8, \mathrm{R}=0.42), \mathrm{CD} 14(p=7-8 \mathrm{e}, \mathrm{R}=0.41)$ in the NF- $\kappa \mathrm{B}$ signaling pathway, $\mathrm{SOCS} 3(p=5 \mathrm{e}-8, \mathrm{R}=$ $0.41)$, MAP2K3 ( $p=2.4 \mathrm{e}-9, \mathrm{R}=0.45), \mathrm{JAK} 3(p=1.7$ $\mathrm{e}-5, \mathrm{R}=0.33)$, IL27RA $(p=5.9 \mathrm{e}-8, \mathrm{R}=0.41)$, and IL13RA1 $(p=3.5$ e-13, $\mathrm{R}=0.53)$ in the JAK/STAT signaling pathway, and USP15 $(p=3.4 \mathrm{e}-11, \mathrm{R}=0.49)$ in the TGF $\beta / \mathrm{SMAD}$ signaling pathway were satisfactory. Therefore, we speculated that these 3 signaling pathways may interact with IKBIP to affect the progression of glioma directly, supporting the view that hsa_circ_0072389, hsa_circ_0072386, hsa_circ_0008621, hsa_circ_0072387, and hsa_circ_0072391 upregulated IKBIP by sponging the adsorption of miR-338-5p, which further promoted glioma through the NF- $\mathrm{B}$ signaling pathway, JAK/STAT signaling pathway, and TGF $\beta / S M A D$ signaling pathway.

In most studies of ceRNAs in tumors, a single circRNA or mRNA could be screened from circRNA chips or
mRNA chips. Then, the targeted miRNA could be predicted easily [31]. However, it was not determined whether a circRNA could only bind to a miRNA invariably [22]. Therefore, this study selected one miRNA, miR-338-5p, as the initial research object and analyzed relationships among circRNAs, miRNAs and mRNAs by exploring the circRNAs to which miR-338$5 p$ may bind. In addition, all genes in 8 common pathways were screened to predict pathways that might be affected by IKBIP. We found that circRNAs (hsa_circ_0072389, hsa_circ_0072386, hsa_circ_0008621, hsa_circ_0072387, and hsa_circ_007239) that bind to miR-338-5p in glioma were all transcribed from the same host gene HMGCS1. In addition, these circRNAs all contained exons 4 and 5 . Through the GEPIA database, we found that the change in HMGCS1 mRNA expression in glioma was not statistically significant when compared with the control group. Therefore, we speculated that pre-RNA splicing of hsa_circ_0072389, hsa_circ_0072386, hsa_circ_0008621, hsa_circ_0072387, and hsa_circ_007239 increased with the progression of glioma. As a large-scale research study based on bioinformatics analysis, this work inevitably lacks sufficient experimental verification, so we need to confirm its authenticity in future research. In addition, due to the lack of glioma data in the GEO and TCGA databases, follow-up data of patients are insufficient. Furthermore, although we revealed that hsa_circ_0072389, hsa_circ_0072386, hsa_circ_0008621, hsa_circ_0072387, and hsa_circ_007239 all originate from the same host gene HMGCS1, limited expression data of circRNAs in glioma prevents us from further investigating differences among these five circRNAs. We suspect that the five circRNAs may be differentially expressed not only in diverse grades or prognostic stages of glioma but also in exosomes, cytoplasm or blood, which play significant roles in different times or spaces. In summary, our study provides a novel idea for the study of the complex network among circRNAs-miRNAsmRNAs, and we also hope to verify the interaction mechanisms among multiple circRNAs, multiple miRNAs, and multiple mRNAs in our future research.

\section{AUTHOR CONTRIBUTIONS}

JL, XL collected and analyzed data, drew plots by $\mathrm{R}$ language and accomplished the manuscript. BZ performed the tests. JL drafted the manuscript. All authors read and approved the final manuscript.

\section{ACKNOWLEDGMENTS}

This work is supported by grants from Science and Technology Program of Shenzhen 
(GJHZ20190821161601670). Thanks Jinan University and Shenzhen People's Hospital for their education and support. Thanks all members for their efforts in this research.

\section{CONFLICTS OF INTEREST}

The authors declare no conflicts of interest related to this study.

\section{REFERENCES}

1. Schwartzbaum JA, Fisher JL, Aldape KD, Wrensch M. Epidemiology and molecular pathology of glioma. Nat Clin Pract Neurol. 2006; 2:494-503. https://doi.org/10.1038/ncpneuro0289 PMID:16932614

2. Van Meir EG, Hadjipanayis CG, Norden AD, Shu HK, Wen PY, Olson JJ. Exciting new advances in neurooncology: the avenue to a cure for malignant glioma. CA Cancer J Clin. 2010; 60:166-93. https://doi.org/10.3322/caac.20069 PMID:20445000

3. Liu J, Jiang X, Zou A, Mai Z, Huang Z, Sun L, Zhao J. circlGHG-Induced Epithelial-to-Mesenchymal Transition Promotes Oral Squamous Cell Carcinoma Progression via miR-142-5p/IGF2BP3 Signaling. Cancer Res. 2021; 81:344-55.

https://doi.org/10.1158/0008-5472.CAN-20-0554 PMID:33203701

4. Qu X, Zheng C, Wang B, Wang F, Sun X, Gao Y, Xia Q, Kong $X$. Comprehensive analysis of circular RNAs from steatotic livers after ischemia and reperfusion injury by next-generation RNA sequencing. FEBS Lett. 2021; 595:99-109.

https://doi.org/10.1002/1873-3468.13960

PMID:33070312

5. Zhao ZJ, Shen J. Circular RNA participates in the carcinogenesis and the malignant behavior of cancer. RNA Biol. 2017; 14:514-21.

https://doi.org/10.1080/15476286.2015.1122162 PMID:26649774

6. Salzman J. Circular RNA Expression: Its Potential Regulation and Function. Trends Genet. 2016; 32:309-16. https://doi.org/10.1016/j.tig.2016.03.002 PMID:27050930

7. Pamudurti NR, Bartok $O$, Jens $M$, Ashwal-Fluss R, Stottmeister C, Ruhe L, Hanan M, Wyler E, PerezHernandez D, Ramberger E, Shenzis S, Samson M, Dittmar G, et al. Translation of CircRNAs. Mol Cell. 2017; 66:9-21.e7. https://doi.org/10.1016/i.molcel.2017.02.021 PMID:28344080
8. Zhong $Y$, Du $Y$, Yang $X$, Mo $Y$, Fan $C$, Xiong $F$, Ren $D$, Ye X, Li C, Wang Y, Wei F, Guo C, Wu X, et al. Circular RNAs function as ceRNAs to regulate and control human cancer progression. Mol Cancer. 2018; 17:79. https://doi.org/10.1186/s12943-018-0827-8 PMID:29626935

9. Peng L, Sang H, Wei S, Li Y, Jin D, Zhu X, Li X, Dang Y, Zhang $G$. circCUL2 regulates gastric cancer malignant transformation and cisplatin resistance by modulating autophagy activation via miR-142-3p/ROCK2. Mol Cancer. 2020; 19:156.

https://doi.org/10.1186/s12943-020-01270-x PMID:33153478

10. Cen J, Liang $Y$, Huang $Y$, Pan $Y$, Shu $G$, Zheng $Z$, Liao $X$, Zhou M, Chen D, Fang Y, Chen W, Luo J, Zhang J. Circular RNA circSDHC serves as a sponge for miR127-3p to promote the proliferation and metastasis of renal cell carcinoma via the CDKN3/E2F1 axis. Mol Cancer. 2021; 20:19.

https://doi.org/10.1186/s12943-021-01314-w PMID:33468140

11. Chen $L Y$, Wang $L$, Ren $Y X$, Pang $Z$, Liu $Y$, Sun XD, Tu J, Zhi Z, Qin Y, Sun LN, Li JM. The circular RNA circERBIN promotes growth and metastasis of colorectal cancer by miR-125a-5p and miR-138-5p/4EBP-1 mediated cap-independent HIF-1 $\alpha$ translation. Mol Cancer. 2020; 19:164.

https://doi.org/10.1186/s12943-020-01272-9 PMID:33225938

12. Du Z, Wang L, Xia Y. Circ_0015756 promotes the progression of ovarian cancer by regulating miR-9425p/CUL4B pathway. Cancer Cell Int. 2020; 20:572. https://doi.org/10.1186/s12935-020-01666-1 PMID:33292255

13. Ou R, Mo L, Tang $H$, Leng $S$, Zhu $H$, Zhao L, Ren $Y, X u Y$. circRNA-AKT1 Sequesters miR-942-5p to Upregulate AKT1 and Promote Cervical Cancer Progression. Mol Ther Nucleic Acids. 2020; 20:308-22. https://doi.org/10.1016/j.omtn.2020.01.003 PMID:32193155

14. Lu J, Wang $Y H$, Huang $X Y$, Xie JW, Wang JB, Lin JX, Chen QY, Cao LL, Huang CM, Zheng CH, Li P. circCEP85L suppresses the proliferation and invasion of gastric cancer by regulating NFKBIA expression via miR-942-5p. J Cell Physiol. 2020; 235:6287-99.

https://doi.org/10.1002/jcp.29556 PMID:32026471

15. Ritchie ME, Phipson B, Wu D, Hu Y, Law CW, Shi W, Smyth GK. limma powers differential expression analyses for RNA-sequencing and microarray studies. Nucleic Acids Res. 2015; 43:e47. https://doi.org/10.1093/nar/gkv007 PMID:25605792 
16. Lewis BP, Burge CB, Bartel DP. Conserved seed pairing, often flanked by adenosines, indicates that thousands of human genes are microRNA targets. Cell. 2005; 120:15-20.

https://doi.org/10.1016/j.cell.2004.12.035

PMID: 15652477

17. Lewis BP, Shih IH, Jones-Rhoades MW, Bartel DP, Burge CB. Prediction of mammalian microRNA targets. Cell. 2003; 115:787-98.

https://doi.org/10.1016/s0092-8674(03)01018-3

PMID:14697198

18. Dudekula DB, Panda AC, Grammatikakis I, De S, Abdelmohsen K, Gorospe M. Circlnteractome: A web tool for exploring circular RNAs and their interacting proteins and microRNAs. RNA Biol. 2016; 13:34-42. https://doi.org/10.1080/15476286.2015.1128065 PMID:26669964

19. Yu G, Wang LG, Han Y, He QY. clusterProfiler: an R package for comparing biological themes among gene clusters. OMICS. 2012; 16:284-87.

https://doi.org/10.1089/omi.2011.0118 PMID:22455463

20. Szklarczyk D, Gable AL, Lyon D, Junge A, Wyder S, Huerta-Cepas J, Simonovic M, Doncheva NT, Morris $\mathrm{JH}$, Bork P, Jensen LJ, Mering CV. STRING v11: proteinprotein association networks with increased coverage, supporting functional discovery in genomewide experimental datasets. Nucleic Acids Res. 2019; 47:D607-13.

https://doi.org/10.1093/nar/gky1131

PMID:30476243

21. Santovito D, Egea V, Bidzhekov K, Natarelli L, Mourão A, Blanchet X, Wichapong K, Aslani M, Brunßen C, Horckmans M, Hristov M, Geerlof A, Lutgens E, et al. Autophagy unleashes noncanonical microRNA functions. Autophagy. 2020; 16:2294-96. https://doi.org/10.1080/15548627.2020.1830523 PMID:33054575

22. Wu P, Li C, Ye DM, Yu K, Li Y, Tang H, Xu G, Yi S, Zhang Z. Circular RNA circEPSTI1 accelerates cervical cancer progression via miR-375/409-3P/515-5p-SLC7A11 axis. Aging (Albany NY). 2021; 13:4663-73.

https://doi.org/10.18632/aging.202518

PMID:33534779

23. Chen $Y$, Yang F, Fang E, Xiao W, Mei H, Li H, Li D, Song $\mathrm{H}$, Wang J, Hong M, Wang X, Huang K, Zheng L, Tong Q. Circular RNA circAGO2 drives cancer progression through facilitating HuR-repressed functions of AGO2-miRNA complexes. Cell Death Differ. 2019; 26:1346-64.

https://doi.org/10.1038/s41418-018-0220-6

PMID:30341421
24. Zhang $X$, Tan $P$, Zhuang $Y$, Du L. hsa_circRNA_001587 upregulates SLC4A4 expression to inhibit migration, invasion, and angiogenesis of pancreatic cancer cells via binding to microRNA-223. Am J Physiol Gastrointest Liver Physiol. 2020; 319:G703-17.

https://doi.org/10.1152/ajpgi.00118.2020 PMID: 32878470

25. Ghetti M, Vannini I, Storlazzi CT, Martinelli G, Simonetti G. Linear and circular PVT1 in hematological malignancies and immune response: two faces of the same coin. Mol Cancer. 2020; 19:69. https://doi.org/10.1186/s12943-020-01187-5 PMID:32228602

26. Sack LM, Davoli T, Li MZ, Li Y, Xu Q, Naxerova K, Wooten EC, Bernardi RJ, Martin TD, Chen T, Leng $Y$, Liang AC, Scorsone KA, et al. Profound Tissue Specificity in Proliferation Control Underlies Cancer Drivers and Aneuploidy Patterns. Cell. 2018; 173:499514.e23.

https://doi.org/10.1016/i.cell.2018.02.037 PMID:29576454

27. Faul F, Erdfelder E, Lang AG, Buchner A. G*Power 3: a flexible statistical power analysis program for the social, behavioral, and biomedical sciences. Behav Res Methods. 2007; 39:175-91.

https://doi.org/10.3758/bf03193146 PMID: $\underline{17695343}$

28. Zhou H, Han L, Wang H, Wei J, Guo Z, Li Z. Chidamide Inhibits Glioma Cells by Increasing Oxidative Stress via the miRNA-338-5p Regulation of Hedgehog Signaling. Oxid Med Cell Longev. 2020; 2020:7126976.

https://doi.org/10.1155/2020/7126976 PMID: 32256960

29. Ma XL, Shang F, Ni W, Zhu J, Luo B, Zhang YQ. MicroRNA-338-5p plays a tumor suppressor role in glioma through inhibition of the MAPK-signaling pathway by binding to FOXD1. J Cancer Res Clin Oncol. 2018; 144:2351-66.

https://doi.org/10.1007/s00432-018-2745-y PMID:30225541

30. Lei D, Zhang F, Yao D, Xiong N, Jiang X, Zhao H. MiR338-5p suppresses proliferation, migration, invasion, and promote apoptosis of glioblastoma cells by directly targeting EFEMP1. Biomed Pharmacother. 2017; 89:957-65.

https://doi.org/10.1016/j.biopha.2017.01.137 PMID:28292024

31. Liang ZZ, Guo C, Zou MM, Meng P, Zhang TT. circRNAmiRNA-mRNA regulatory network in human lung cancer: an update. Cancer Cell Int. 2020; 20:173. https://doi.org/10.1186/s12935-020-01245-4 PMID: $\underline{32467668}$ 


\section{SUPPLEMENTARY MATERIALS}

\section{Supplementary Figures}

A

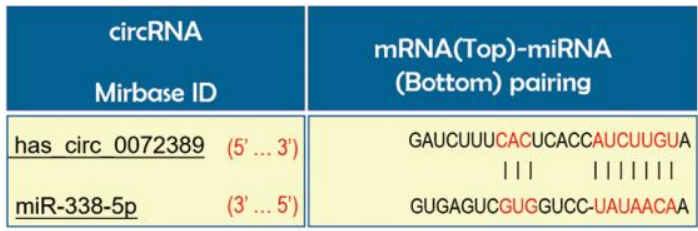

C

\begin{tabular}{|c|c|}
\hline $\begin{array}{c}\text { circRNA } \\
\text { Mirbase ID }\end{array}$ & $\begin{array}{l}\text { mRNA(Top)-miRNA } \\
\text { (Bottom) pairing }\end{array}$ \\
\hline has circ $0072386 \quad\left(5^{\prime} \ldots 3^{\prime}\right)$ & $\begin{array}{l}\text { GAUCUUUCACUCACCAUCUUGUA } \\
\|\|\|\quad\|\|\|\|\|\end{array}$ \\
\hline miR-338-5p & GUGAGUCGUGGUCC-UAUAACAA \\
\hline
\end{tabular}

E

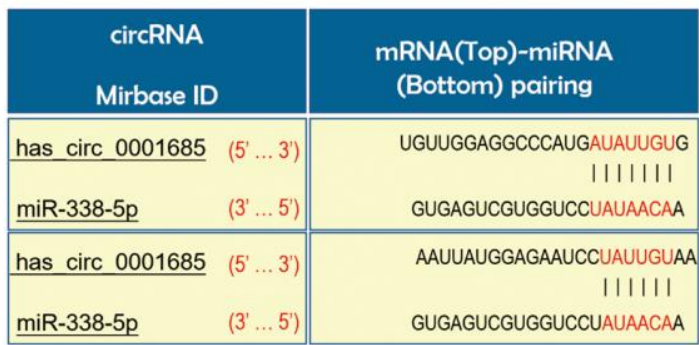

B

\begin{tabular}{|c|c|}
\hline $\begin{array}{c}\text { circRNA } \\
\text { Mirbase ID }\end{array}$ & $\begin{array}{l}\text { mRNA(Top)-miRNA } \\
\text { (Bottom) pairing }\end{array}$ \\
\hline has circ $0072387 \quad\left(5^{\prime} \ldots 3^{\prime}\right)$ & $\begin{array}{c}\text { GAUCUUUCACUCACCAUCUUGUA } \\
\|\| \|\end{array}$ \\
\hline miR-338-5p & GUGAGUCGUGGUCC-UAUAACAA \\
\hline
\end{tabular}

D

\begin{tabular}{|c|c|}
\hline $\begin{array}{c}\text { circRNA } \\
\text { Mirbase ID }\end{array}$ & $\begin{array}{l}\text { mRNA(Top)-miRNA } \\
\text { (Bottom) pairing }\end{array}$ \\
\hline has circ $0008621 \quad\left(5^{\prime} \ldots 3^{\prime}\right)$ & GAUCUUUCACUCACCAUCUUGUA \\
\hline miR-338-5p $\quad\left(3^{\prime} \ldots 5^{\prime}\right)$ & $\begin{array}{c}11111111111 \\
\text { GUGAGUCGUGGUCC-UAUAACAA }\end{array}$ \\
\hline
\end{tabular}

$\mathbf{F}$

\begin{tabular}{|c|c|}
\hline $\begin{array}{c}\text { circRNA } \\
\text { Mirbase ID }\end{array}$ & $\begin{array}{l}\text { mRNA(Top)-miRNA } \\
\text { (Bottom) pairing }\end{array}$ \\
\hline has circ $0044234 \quad\left(5^{\prime} \ldots 3^{\prime}\right)$ & $\begin{array}{r}\text { AGAAAAGCCAUGAUGAUAUUGUU } \\
1\|1\|\|\|\end{array}$ \\
\hline miR-338-5p & GUGAGUCGUGGUCCUAUAACAA \\
\hline
\end{tabular}

G

\begin{tabular}{|c|c|}
\hline $\begin{array}{c}\text { circRNA } \\
\text { Mirbase ID }\end{array}$ & $\begin{array}{l}\text { mRNA(Top)-miRNA } \\
\text { (Bottom) pairing }\end{array}$ \\
\hline has circ $0072391 \quad\left(5^{\prime} \ldots 3^{\prime}\right)$ & $\begin{array}{c}\text { GAUCUUUCACUCACCAUCUUGUA } \\
\|1\| \quad\|1\| 1 \|\end{array}$ \\
\hline miR-338-5p & GUGAGUCGUGGUCC-UAUAACAA \\
\hline
\end{tabular}

H

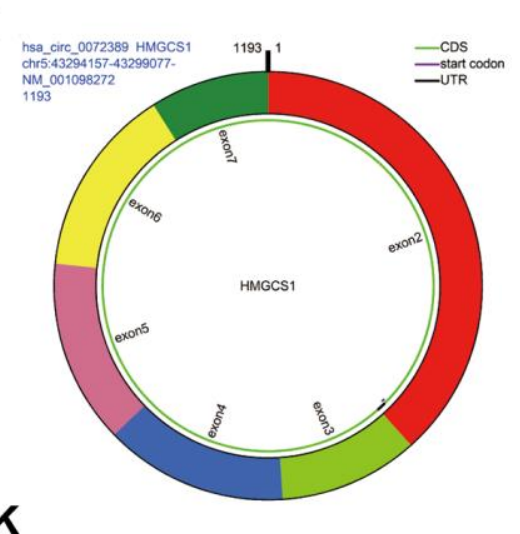

K

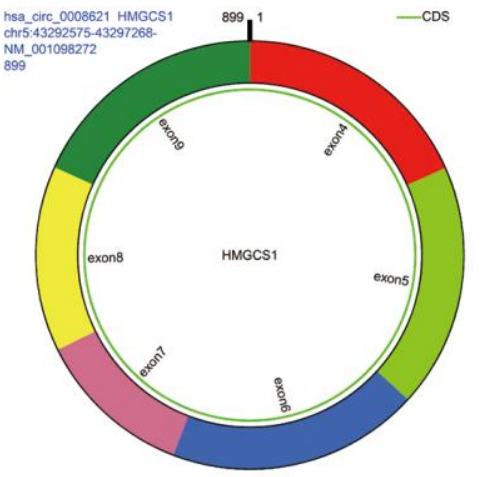

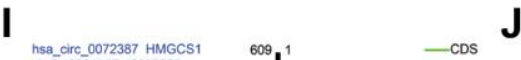
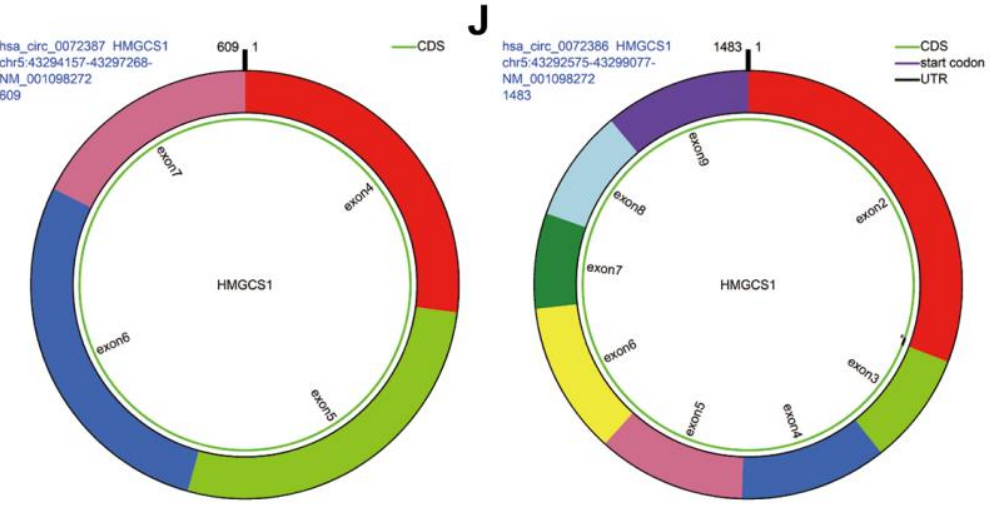

$\mathbf{L}$

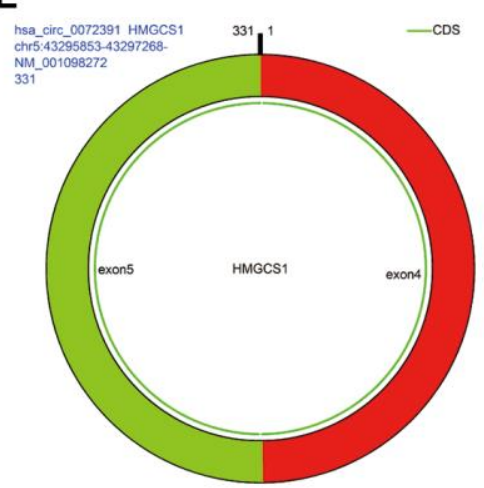

Supplementary Figure 1. (A-G) Binding site between hsa_circ_0072389, hsa_circ_0072386, hsa_circ_0008621, hsa_circ_0072387, hsa_circ_0072391, hsa_circ_0044234, hsa_circ_0001685 with miR-338-5p. (H-L) Exon composition, host gene information, chromosome location information of hsa_circ_0072389, hsa_circ_0072386, hsa_circ_0008621, hsa_circ_0072387, hsa_circ_0072391. 
hsa_circ_0072389

CTCTTTCACCATGCCTGGATCACTTCCTTTGAATGCAGAAGCTTGCTGGCCAAAAGATGTGGGAATTGTTGCCCTTGAGATCTATTTTCCTTCTCAATATGTTGATCAAGCAGAG TTGGAAAAATATGATGGTGTAGATGCTGGAAAGTATACCATTGGCTTGGGCCAGGCCAAGATGGGCTTCTGCACAGATAGAGAAGATATTAACTCTCTTTGCATGACTGTGGT TCAGAATCTTATGGAGAGAAATAACCTTTCCTATGATTGCATTGGGCGGCTGGAAGTTGGAACAGAGACAATCATCGACAAATCAAAGTCTGTGAAGACTAATTTGATGCAGC TGTTTGAAGAGTCTGGGAATACAGATATAGAAGGAATCGACACAACTAATGCATGCTATGGAGGCACAGCTGCTGTCTTCAATGCTGTTAACTGGATTGAGTCCAGCTCTTGG GATGGACGGTATGCCCTGGTAGTTGCAGGAGATATTGCTGTATATGCCACAGGAAATGCTAGACCTACAGGTGGAGTTGGAGCAGTAGCTCTGCTAATTGGGCCAAATGCTC CTTTAATTTTTGAACGAGGGCTTCGTGGGACACATATGCAACATGCCTATGATTTTTACAAGCCTGATATGCTATCTGAATATCCTATAGTAGATGGAAAACTCTCCATACAGTG CTACCTCAGTGCATTAGACCGCTGCTATTCTGTCTACTGCAAAAAGATCCATGCCCAGTGGCAGAAAGAGGGAAATGATAAAGATTTTACCTTGAATGATTTTGGCTTCATGAT CTTTCACTCACCATATTGTAAACTGGTTCAGAAATCTCTAGCTCGGATGTTGCTGAATGACTTCCTTAATGACCAGAATAGAGATAAAAATAGTATCTATAGTGGCCTGGAAGC CTTTGGGGATGTTAAATTAGAAGACACCTACTTTGATAGAGATGTGGAGAAGGCATTTATGAAGGCTAGCTCTGAACTCTTCAGTCAGAAAACAAAGGCATCTTTACTTGTATC AAATCAAAATGGAAATATGTACACATCTTCAGTATATGGTTCCCTTGCATCTGTTCTAGCACAGTACTCACCTCAGCAATTAGCAGGGAAGAGAATTGGAGTGTTTTCTTATGGT TCTGGTTTGGCTGCCACTCTGTACTCTCTTAAAGTCACACAAGATGCTACACCGG

hsa_circ_0072387

GGCTTCGTGGGACACATATGCAACATGCCTATGATTTTTACAAGCCTGATATGCTATCTGAATATCCTATAGTAGATGGAAAACTCTCCATACAGTGCTACCTCAGTGCATTAGA CCGCTGCTATTCTGTCTACTGCAAAAAGATCCATGCCCAGTGGCAGAAAGAGGGAAATGATAAAGATTTTACCTTGAATGATTTTGGCTTCATGATCTTTCACTCACCATATTGT AAACTGGTTCAGAAATCTCTAGCTCGGATGTTGCTGAATGACTTCCTTAATGACCAGAATAGAGATAAAAATAGTATCTATAGTGGCCTGGAAGCCTTTGGGGATGTTAAATTA GAAGACACCTACTTTGATAGAGATGTGGAGAAGGCATTTATGAAGGCTAGCTCTGAACTCTTCAGTCAGAAAACAAAGGCATCTTTACTTGTATCAAATCAAAATGGAAATAT GTACACATCTTCAGTATATGGTTCCCTTGCATCTGTTCTAGCACAGTACTCACCTCAGCAATTAGCAGGGAAGAGAATTGGAGTGTTTTCTTATGGTTCTGGTTTGGCTGCCACT CTGTACTCTCTTAAAGTCACACAAGATGCTACACCGG

hsa_circ_0072386

CTCTTTCACCATGCCTGGATCACTTCCTTTGAATGCAGAAGCTTGCTGGCCAAAAGATGTGGGAATTGTTGCCCTTGAGATCTATTTTCCTTCTCAATATGTTGATCAAGCAGAG TTGGAAAAATATGATGGTGTAGATGCTGGAAAGTATACCATTGGCTTGGGCCAGGCCAAGATGGGCTTCTGCACAGATAGAGAAGATATTAACTCTCTTTGCATGACTGTGGT TCAGAATCTTATGGAGAGAAATAACCTTTCCTATGATTGCATTGGGCGGCTGGAAGTTGGAACAGAGACAATCATCGACAAATCAAAGTCTGTGAAGACTAATTTGATGCAGC TGTTTGAAGAGTCTGGGAATACAGATATAGAAGGAATCGACACAACTAATGCATGCTATGGAGGCACAGCTGCTGTCTTCAATGCTGTTAACTGGATTGAGTCCAGCTCTTGG GATGGACGGTATGCCCTGGTAGTTGCAGGAGATATTGCTGTATATGCCACAGGAAATGCTAGACCTACAGGTGGAGTTGGAGCAGTAGCTCTGCTAATTGGGCCAAATGCTC CTTTAATTTTTGAACGAGGGCTTCGTGGGACACATATGCAACATGCCTATGATTTTTACAAGCCTGATATGCTATCTGAATATCCTATAGTAGATGGAAAACTCTCCATACAGTG CTACCTCAGTGCATTAGACCGCTGCTATTCTGTCTACTGCAAAAAGATCCATGCCCAGTGGCAGAAAGAGGGAAATGATAAAGATTTTACCTTGAATGATTTTGGCTTCATGAT CTTTCACTCACCATATTGTAAACTGGTTCAGAAATCTCTAGCTCGGATGTTGCTGAATGACTTCCTTAATGACCAGAATAGAGATAAAAATAGTATCTATAGTGGCCTGGAAGC CTTTGGGGATGTTAAATTAGAAGACACCTACTTTGATAGAGATGTGGAGAAGGCATTTATGAAGGCTAGCTCTGAACTCTTCAGTCAGAAAACAAAGGCATCTTTACTTGTATC AAATCAAAATGGAAATATGTACACATCTTCAGTATATGGTTCCCTTGCATCTGTTCTAGCACAGTACTCACCTCAGCAATTAGCAGGGAAGAGAATTGGAGTGTTTTCTTATGGT TCTGGTTTGGCTGCCACTCTGTACTCTCTTAAAGTCACACAAGATGCTACACCGGGGTCTGCTCTTGATAAAATAACAGCAAGTTTATGTGATCTTAAATCAAGGCTTGATTCAA GAACTGGTGTGGCACCAGATGTCTTCGCTGAAAACATGAAGCTCAGAGAGGACACCCATCATTTGGTCAACTATATTCCCCAGGGTTCAATAGATTCACTCTTTGAAGGAACG TGGTACTTAGTTAGGGTGGATGAAAAGCACAGAAGAACTTACGCTCGGCGTCCCACTCCAAATGATGACACTTTGGATGAAGGAGTAGGACTTGTGCATTCAAACATAGCAA CTGAG

hsa_circ_0008621

ACCTCAGTGCATTAGACCGCTGCTATTCTGTCTACTGCAAAAAGATCCATGCCCAGTGGCAGAAAGAGGGAAATGATAAAGATTTTACCTTGAATGATTTTGGCTTCATGATCT TTCACTCACCATATTGTAAACTGGTTCAGAAATCTCTAGCTCGGATGTTGCTGAATGACTTCCTTAATGACCAGAATAGAGATAAAAATAGTATCTATAGTGGCCTGGAAGCCTT TGGGGATGTTAAATTAGAAGACACCTACTTTGATAGAGATGTGGAGAAGGCATTTATGAAGGCTAGCTCTGAACTCTTCAGTCAGAAAACAAAGGCATCTTTACTTGTATCAA ATCAAAATGGAAATATGTACACATCTTCAGTATATGGTTCCCTTGCATCTGTTCTAGCACAGTACTCACCTCAGCAATTAGCAGGGAAGAGAATTGGAGTGTTTTCTTATGGTTC TGGTTTGGCTGCCACTCTGTACTCTCTTAAAGTCACACAAGATGCTACACCGGGGTCTGCTCTTGATAAAATAACAGCAAGTTTATGTGATCTTAAATCAAGGCTTGATTCAAGA ACTGGTGTGGCACCAGATGTCTTCGCTGAAAACATGAAGCTCAGAGAGGACACCCATCATTTGGTCAACTATATTCCCCAGGGTTCAATAGATTCACTCTTTGAAGGAACGTG GTACTTAGTTAGGGTGGATGAAAAGCACAGAAGAACTTACGCTCGGCGTCCCACTCCAAATGATGACACTTTGGATGAAGGAGTAGGACTTGTGCATTCAAACATAGCAACT GAG

hsa_circ_0072391

GGCTTCGTGGGACACATATGCAACATGCCTATGATTTTTACAAGCCTGATATGCTATCTGAATATCCTATAGTAGATGGAAAACTCTCCATACAGTGCTACCTCAGTGCATTAGA CCGCTGCTATTCTGTCTACTGCAAAAAGATCCATGCCCAGTGGCAGAAAGAGGGAAATGATAAAGATTTTACCTTGAATGATTTTGGCTTCATGATCTTTCACTCACCATATTGT AAACTGGTTCAGAAATCTCTAGCTCGGATGTTGCTGAATGACTTCCTTAATGACCAGAATAGAGATAAAAATAGTATCTATAGTGGCCTGGAAGCCTTTGG

Supplementary Figure 2. The sequence of hsa_circ_0072389, hsa_circ_0072387, hsa_circ_0072386, hsa_circ_0008621, hsa_circ_0072391. 

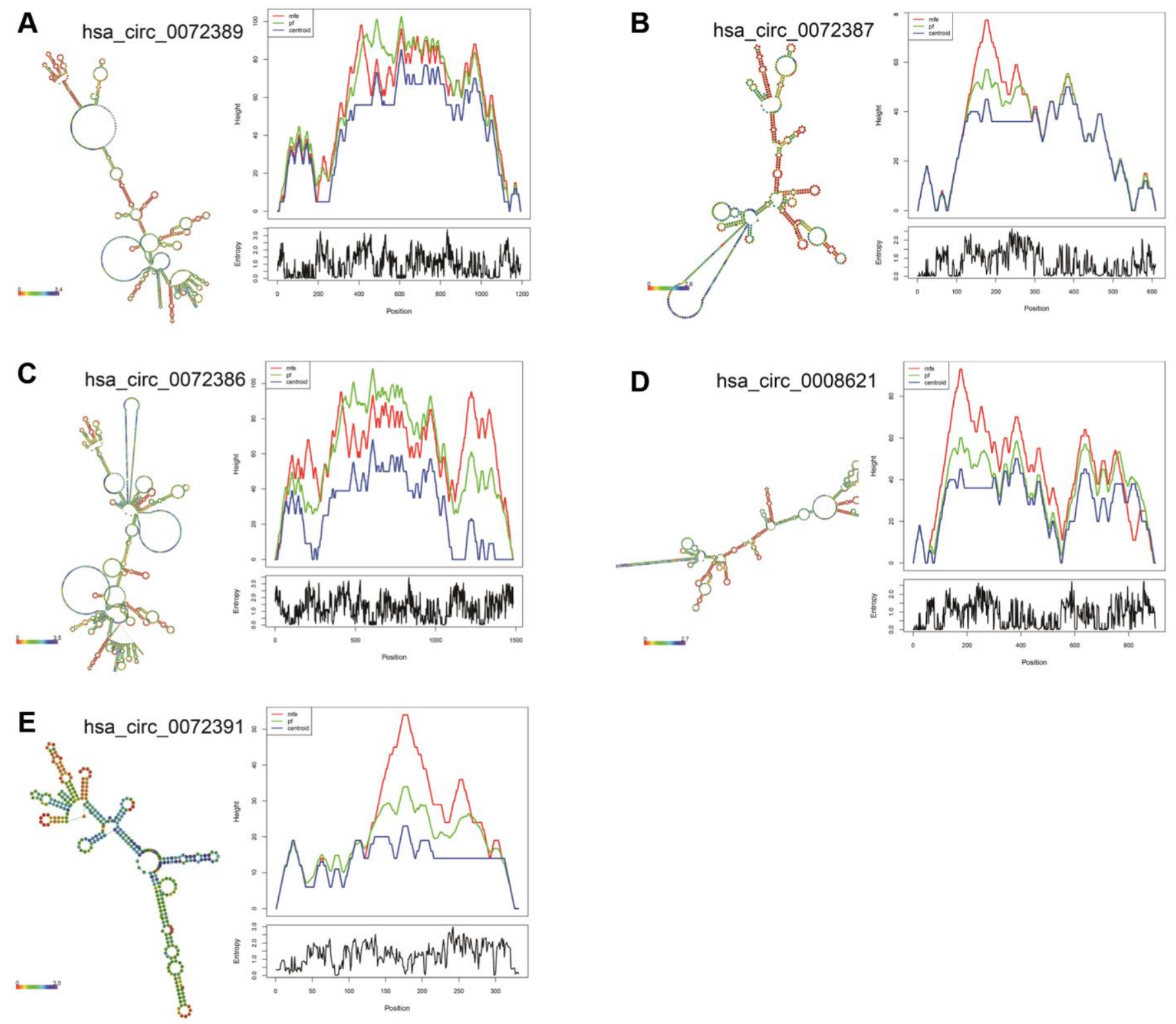

Supplementary Figure 3. The secondary structure and minimum free energy (MFE) structure of (A) hsa_circ_0072389, (B) hsa_circ_0072387, (C) hsa_circ_0072386, (D) hsa_circ_0008621, (E) hsa_circ_0072391. 
A

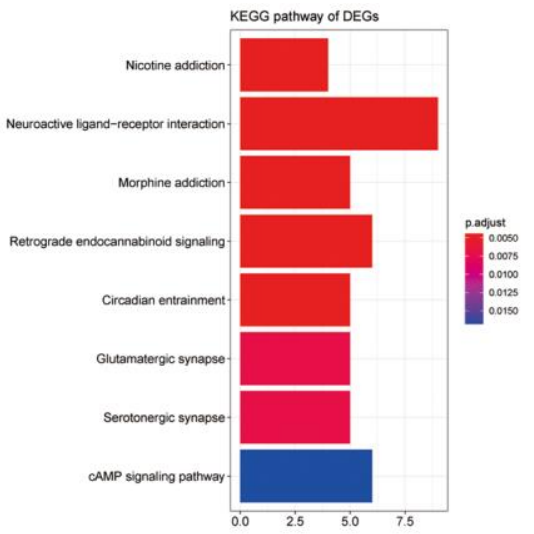

C

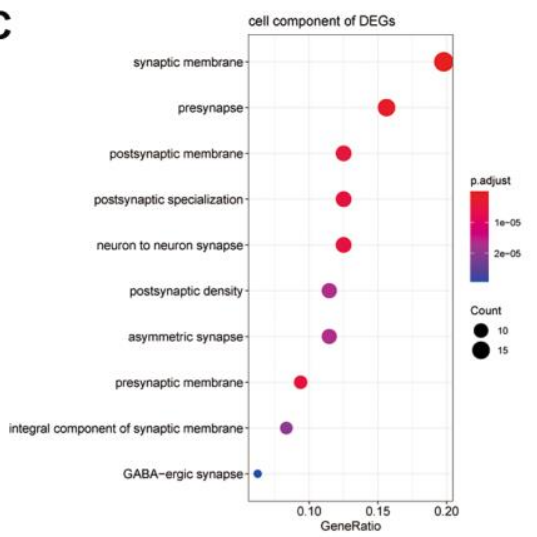

E

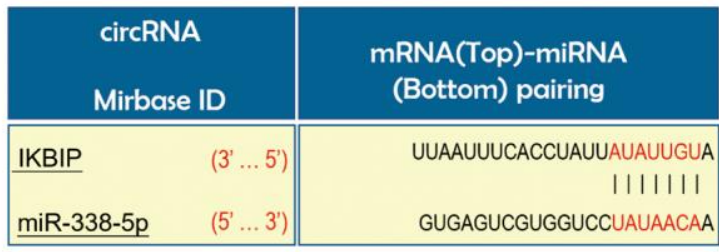

G

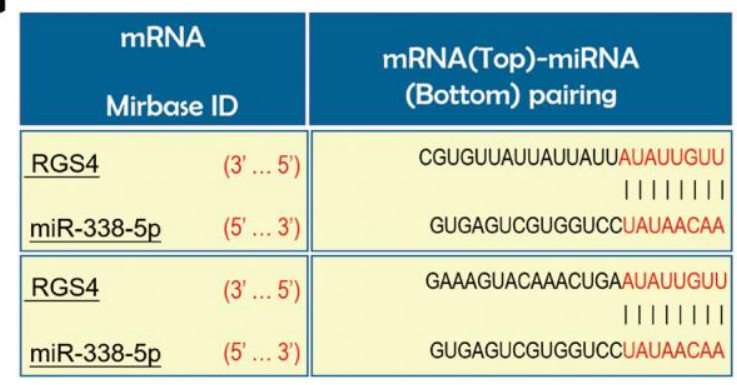

B

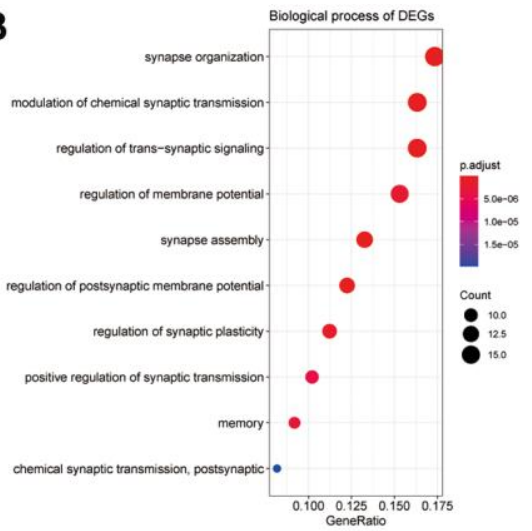

D

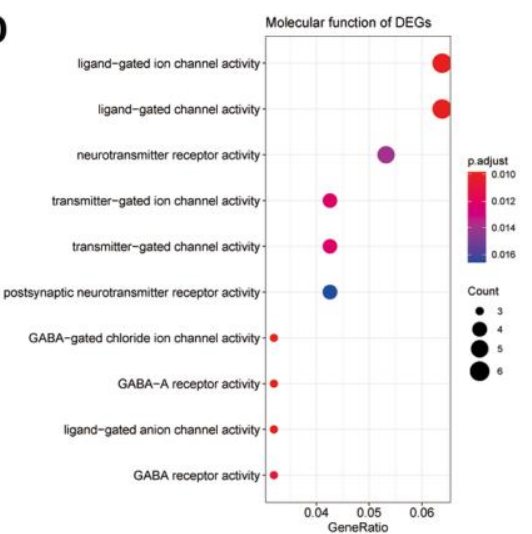

F

\begin{tabular}{|c|c|c|}
\multicolumn{2}{|c|}{ mRNA } & \multicolumn{1}{c|}{$\begin{array}{c}\text { mRNA(Top)-miRNA } \\
\text { Mirbase ID }\end{array}$} \\
\hline (Bottom) pairing
\end{tabular}

H

\begin{tabular}{|c|c|c|}
\hline \multicolumn{2}{|c|}{$\begin{array}{l}\text { mRNA } \\
\text { Mirbase ID }\end{array}$} & $\begin{array}{l}\text { mRNA(Top)-miRNA } \\
\text { (Bottom) pairing }\end{array}$ \\
\hline NRG1 & $\left(3^{\prime} \ldots 5^{\prime}\right)$ & $\begin{array}{r}\text { UCAUCUCCAGUGAGCAUAUUGUU } \\
\qquad||||||||\end{array}$ \\
\hline miR-338-5p & $\left(5^{\prime} \ldots 3^{\prime}\right)$ & GUGAGUCGUGGUCCUAUAACAA \\
\hline NRG1 & $\left(3^{\prime} \ldots 5^{\prime}\right)$ & $\begin{array}{r}\text { UUCUUUUGUAAAUACAUAUUGUA } \\
\qquad||||||\end{array}$ \\
\hline miR-338-5p & $\left(5^{\prime} \ldots 3^{\prime}\right)$ & GUGAGUCGUGGUCCUAUAACAA \\
\hline
\end{tabular}

Supplementary Figure 4. (A) Pathways involved in the 100 mRNAs analysis by KEGG. (B-D) Biological process, cell component, and molecular function of the 100 mRNAs by GO analysis. (E-H) Binding site between CCBE1, IKBIP, NRG1, and RGS4 with miR-338-5p. 


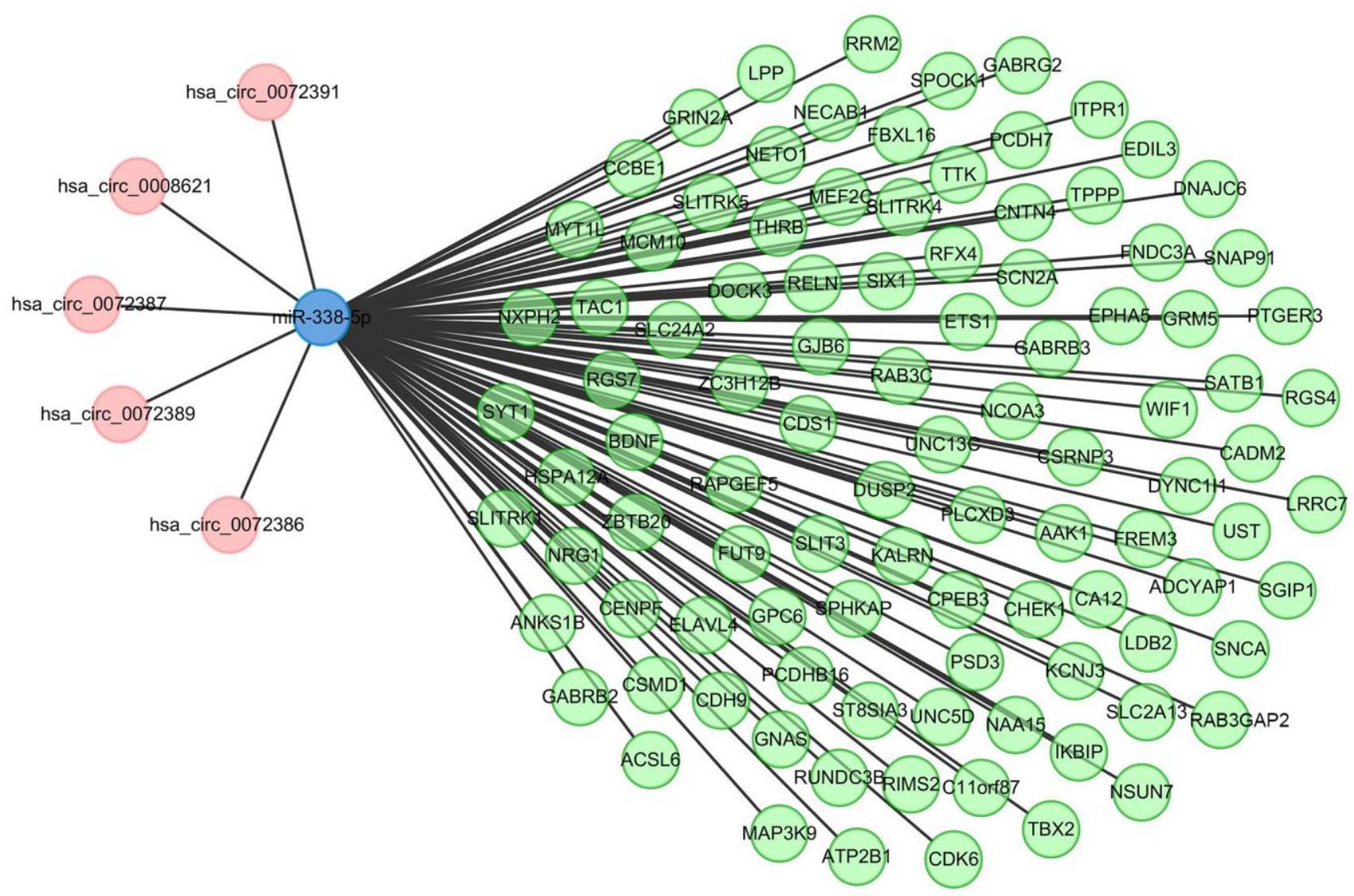

Supplementary Figure 5. circRNA-miRNA-mRNA network about hsa_circ_0072389, hsa_circ_0072386, hsa_circ_0008621, hsa_circ_0072387, hsa_circ_0072391, miR-338-5p, and the 100 mRNAs that might bind to miR-338-5p. 


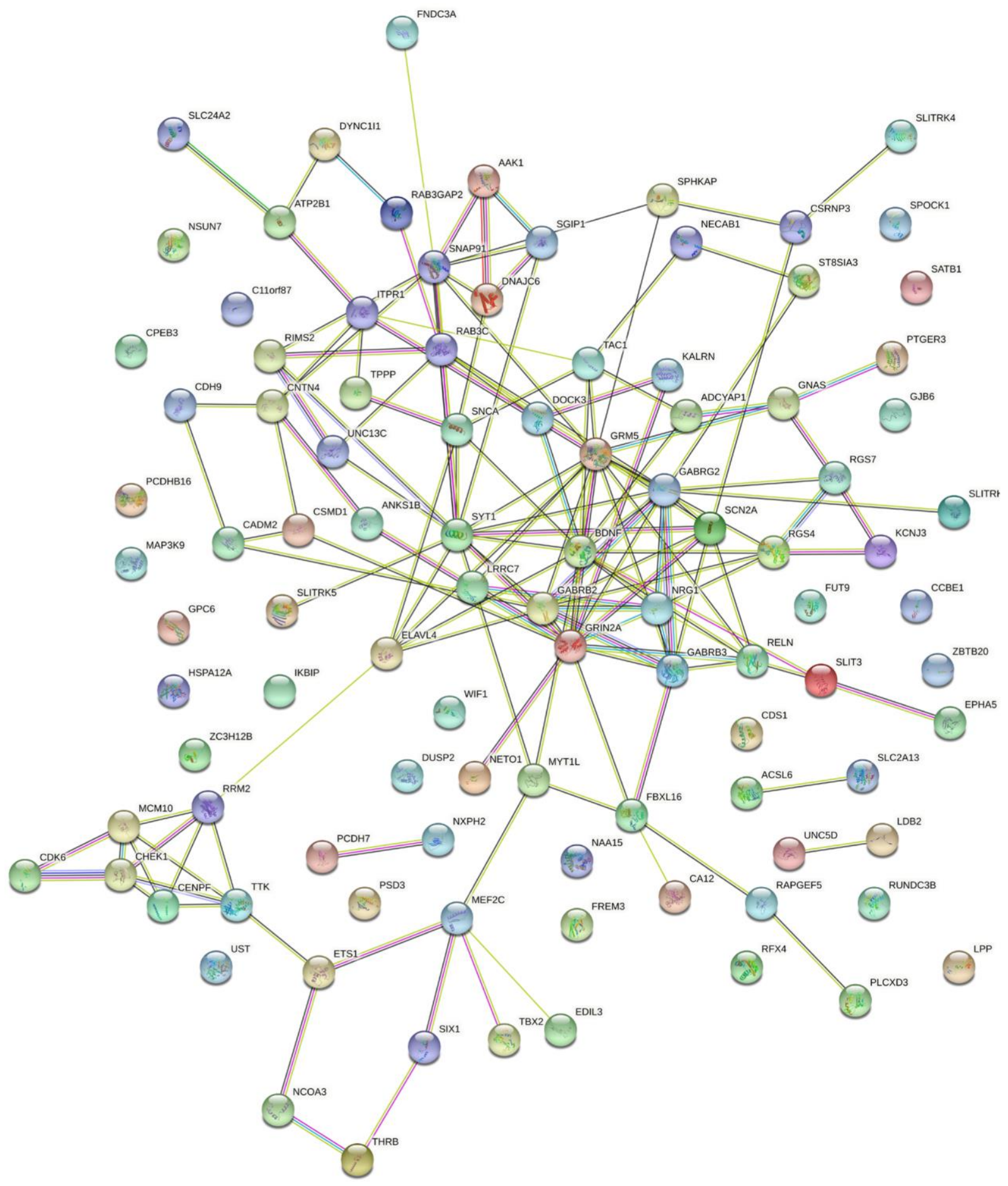

Supplementary Figure 6. PPI network about 100 mRNAs that might bind to miR-338-5p. 


\section{Hedgehog signaling pathway}
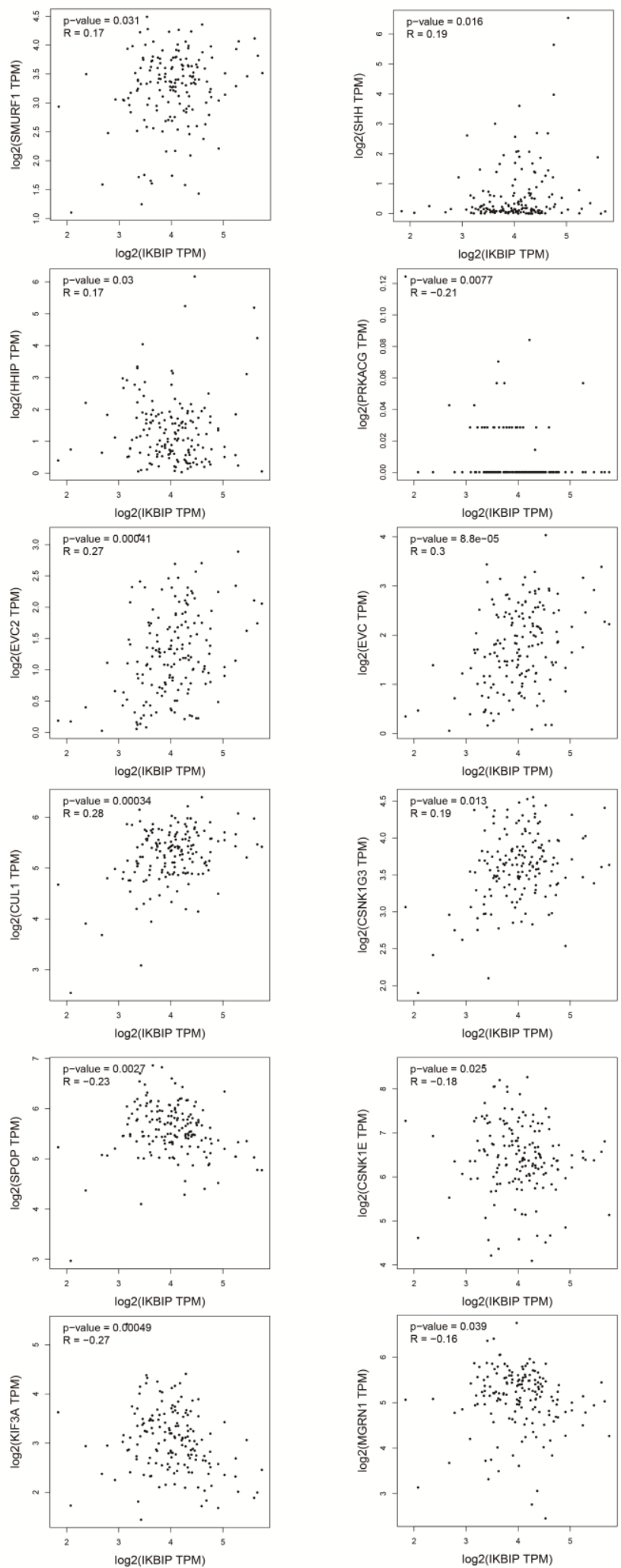
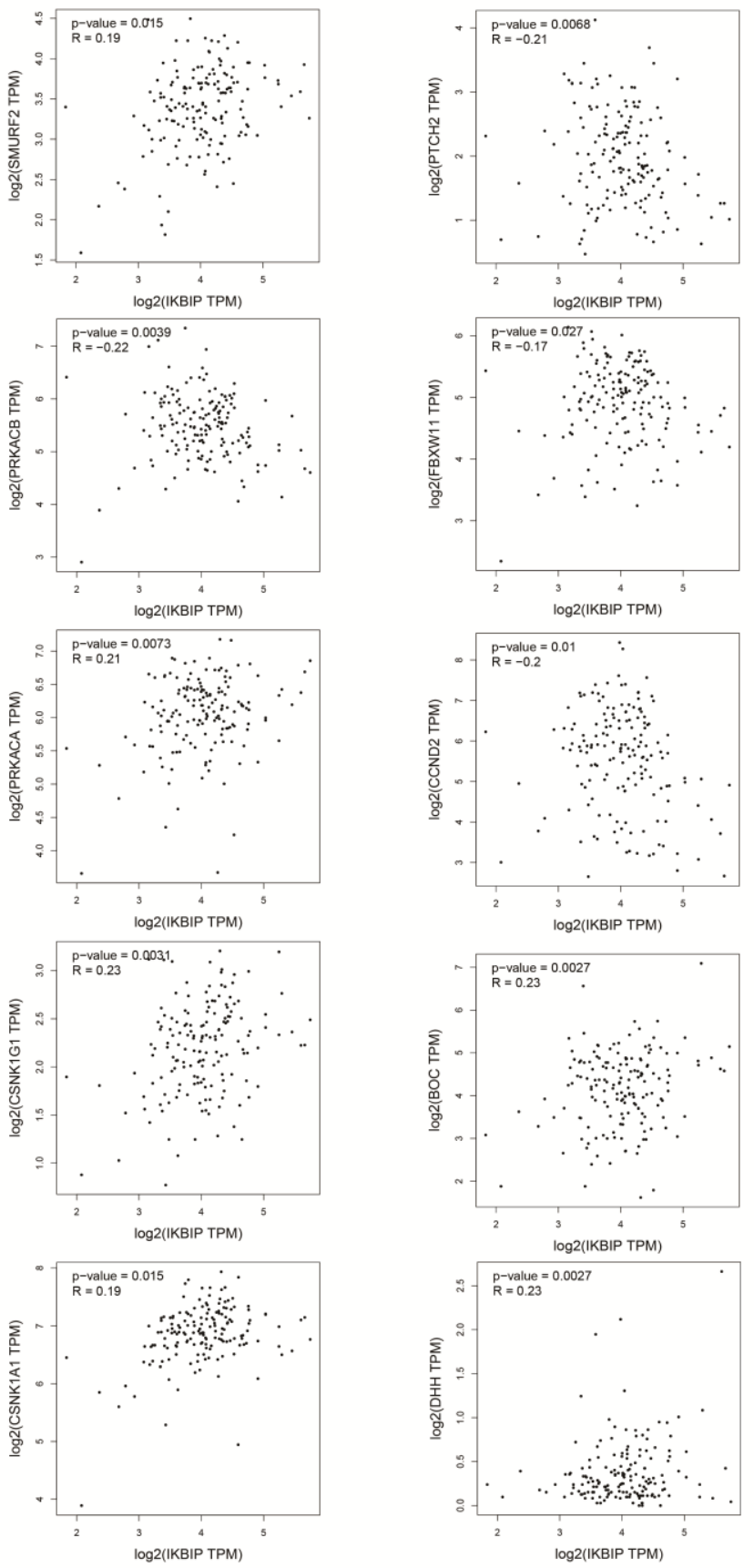

Supplementary Figure 7. Scatter plot shown genes in Hedgehog signaling pathway related to IKBIP expression in glioma. 


\section{JAK/STAT signaling pathway}
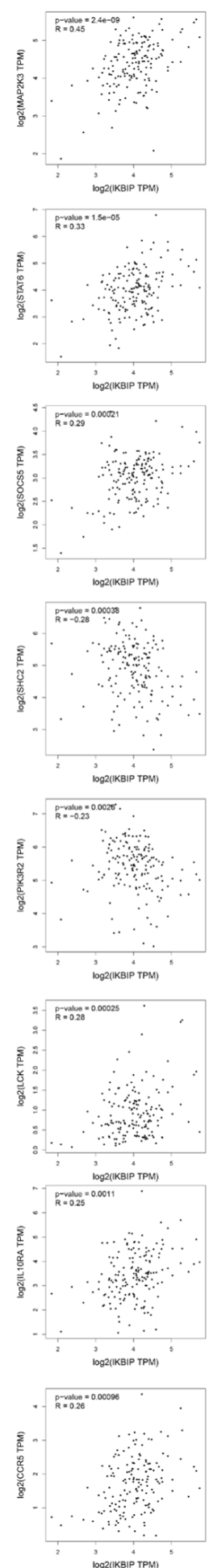
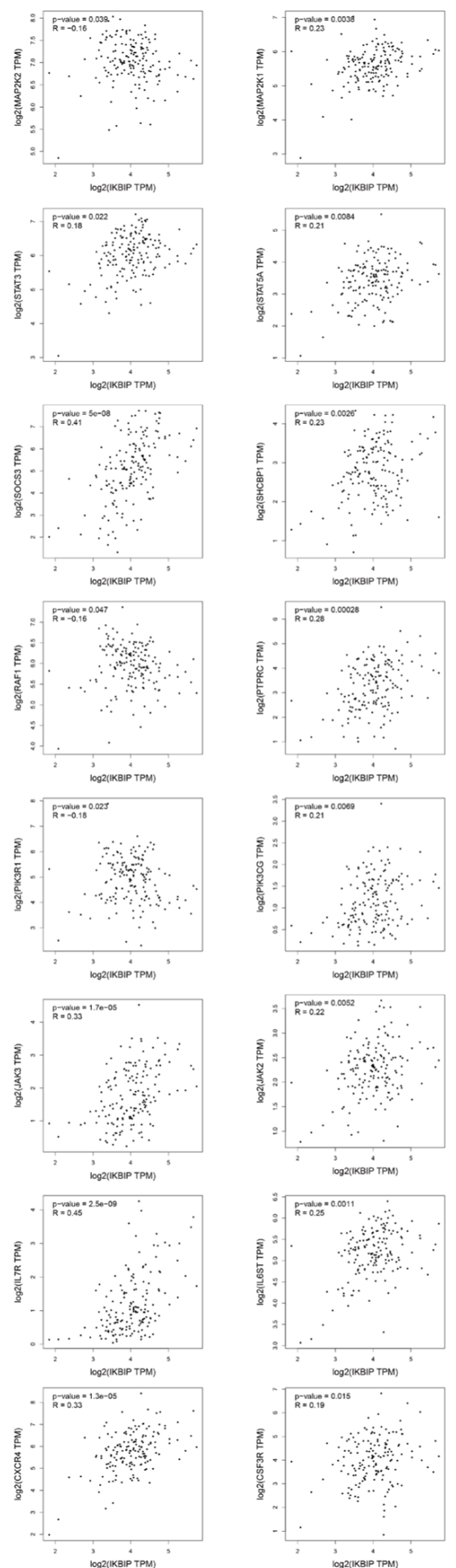
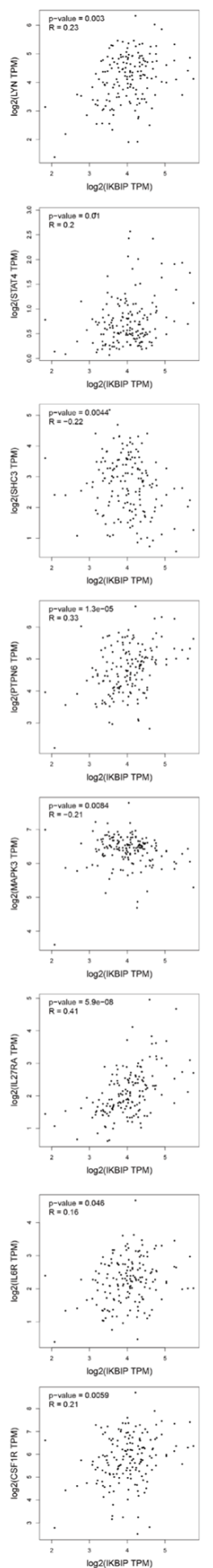
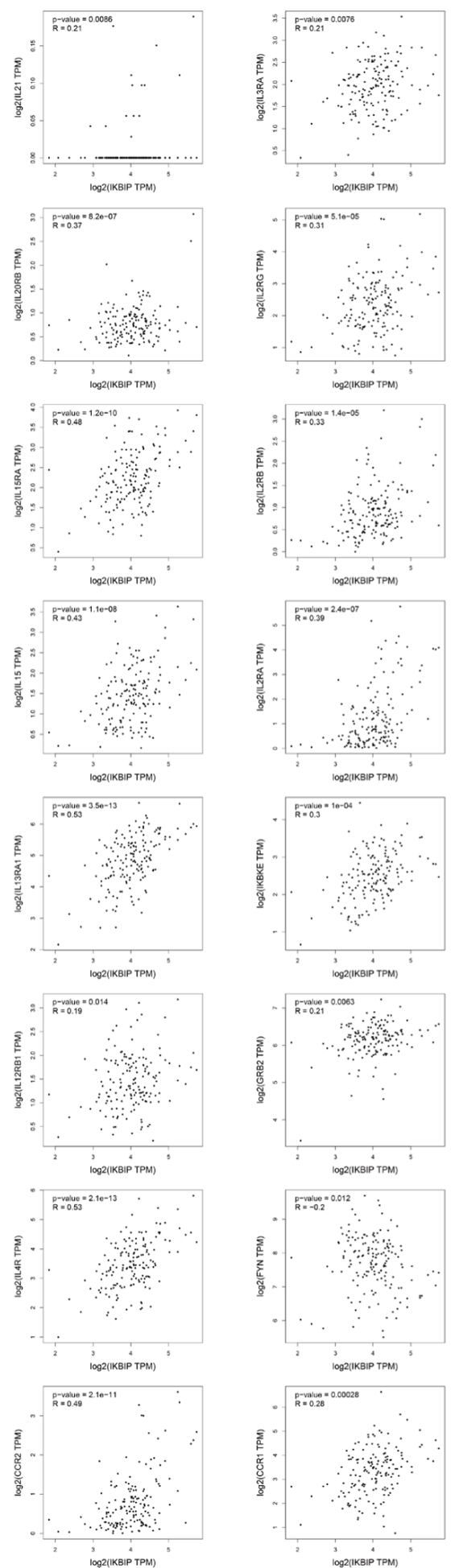

Supplementary Figure 8. Scatter plot shown genes in JAK/STAT signaling pathway related to IKBIP expression in glioma. 


\section{MAPK signaling pathway}
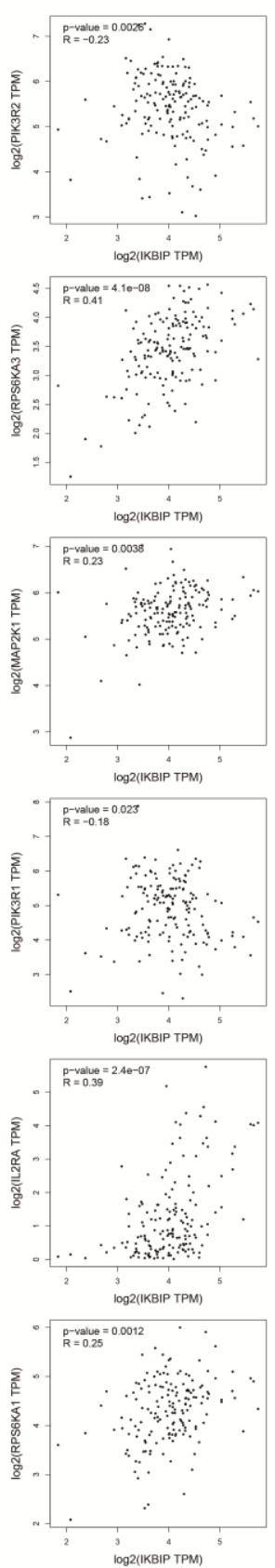
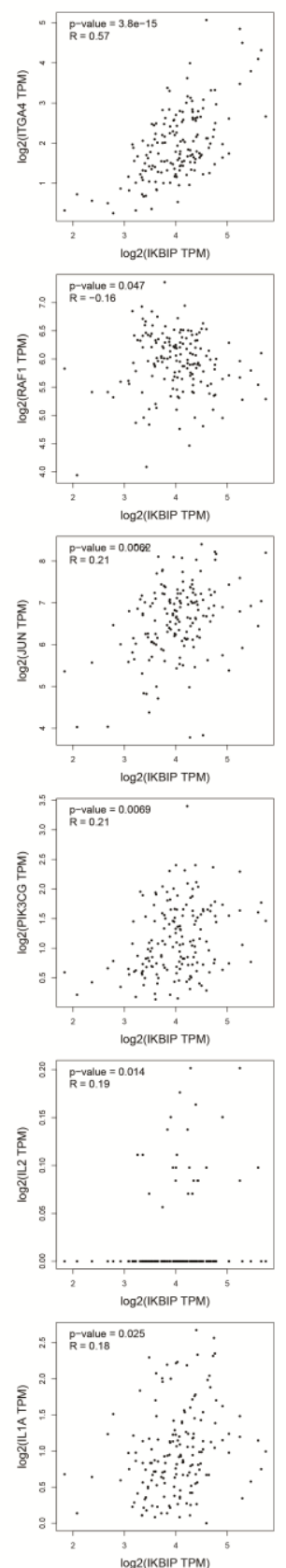
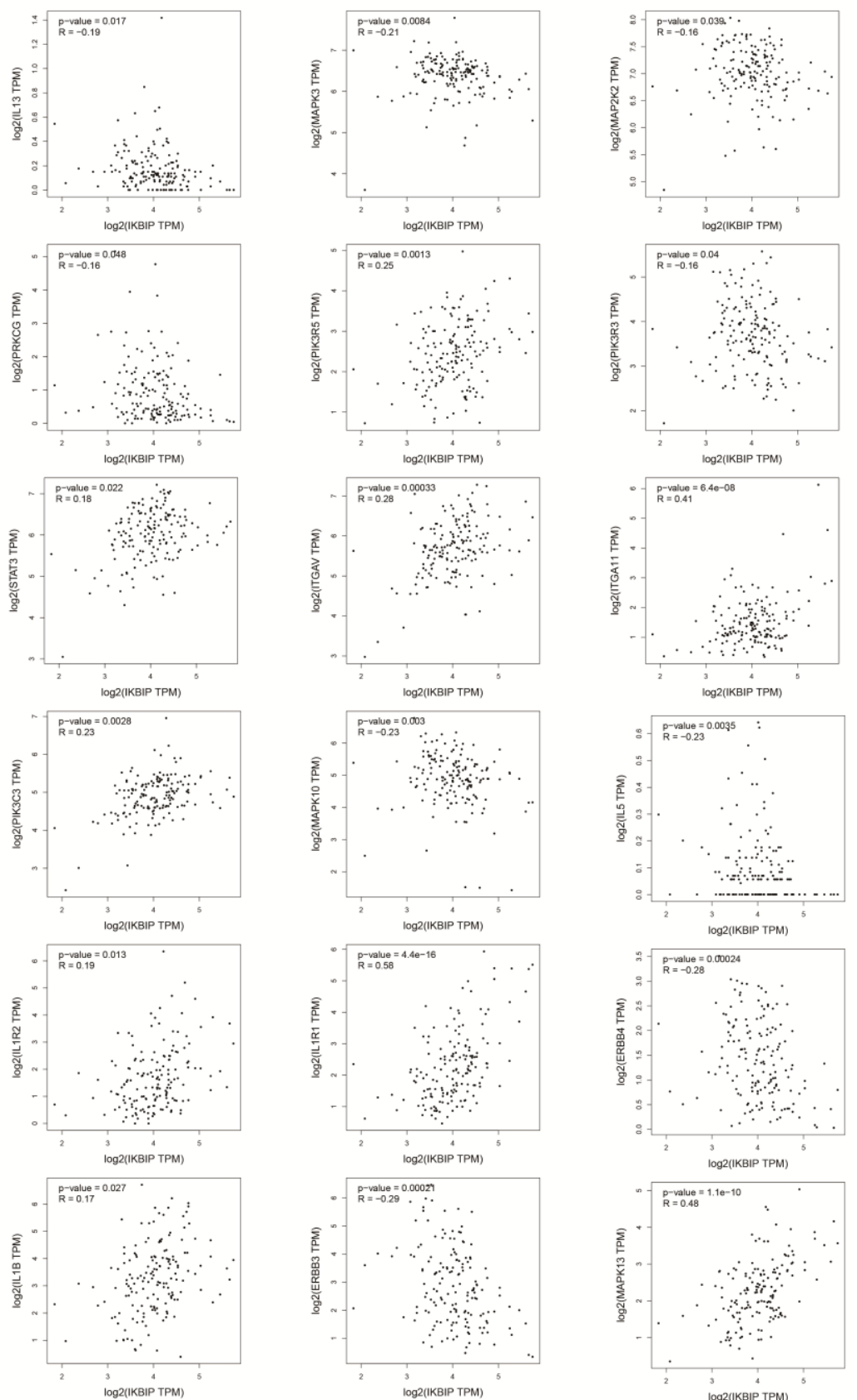

Supplementary Figure 9. Scatter plot shown genes in MAPK signaling pathway related to IKBIP expression in glioma. 
NF-KB signaling pathway

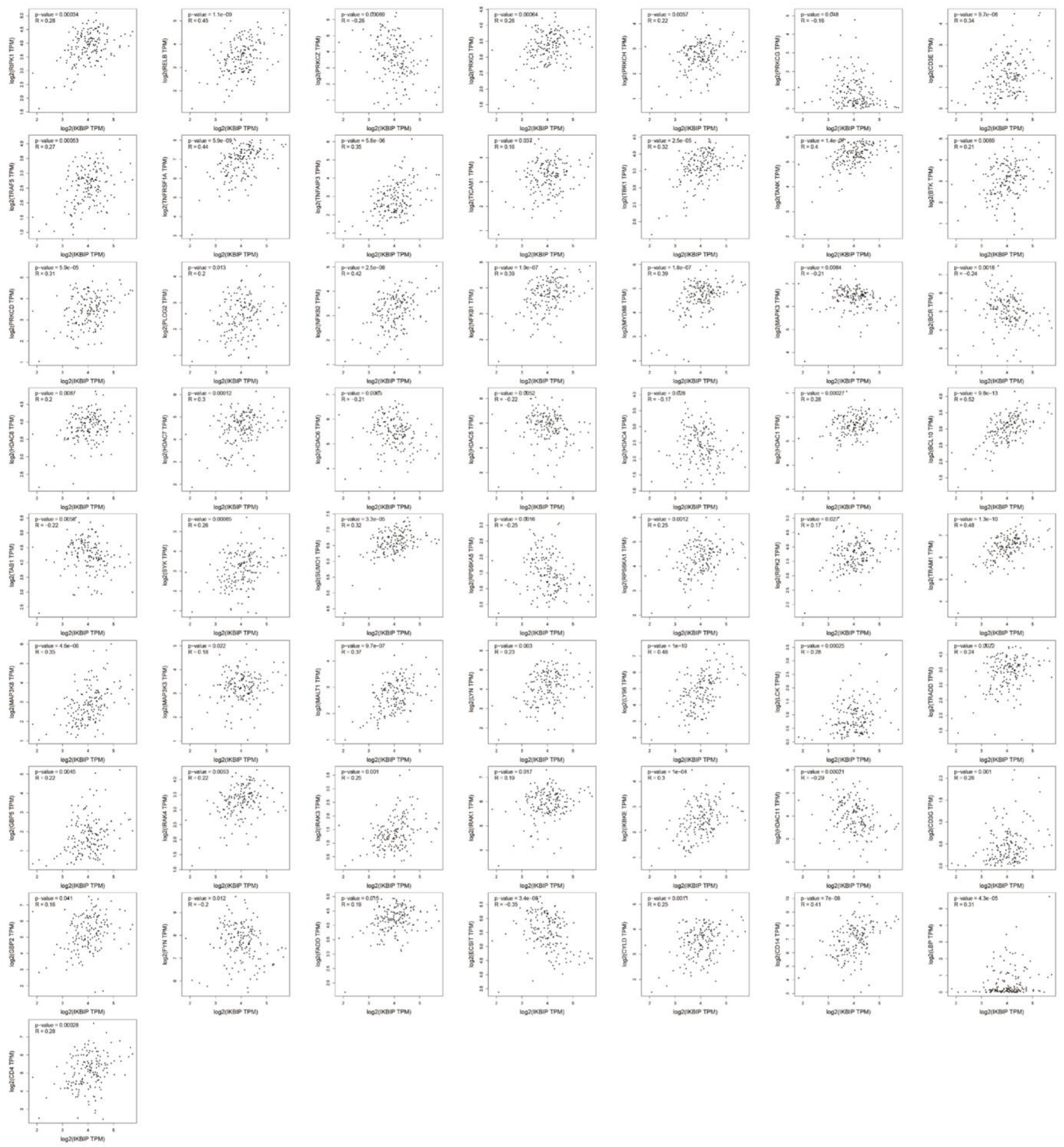

Supplementary Figure 10. Scatter plot shown genes in NF-KB signaling pathway related to IKBIP expression in glioma. 


\section{Notch signaling pathway}
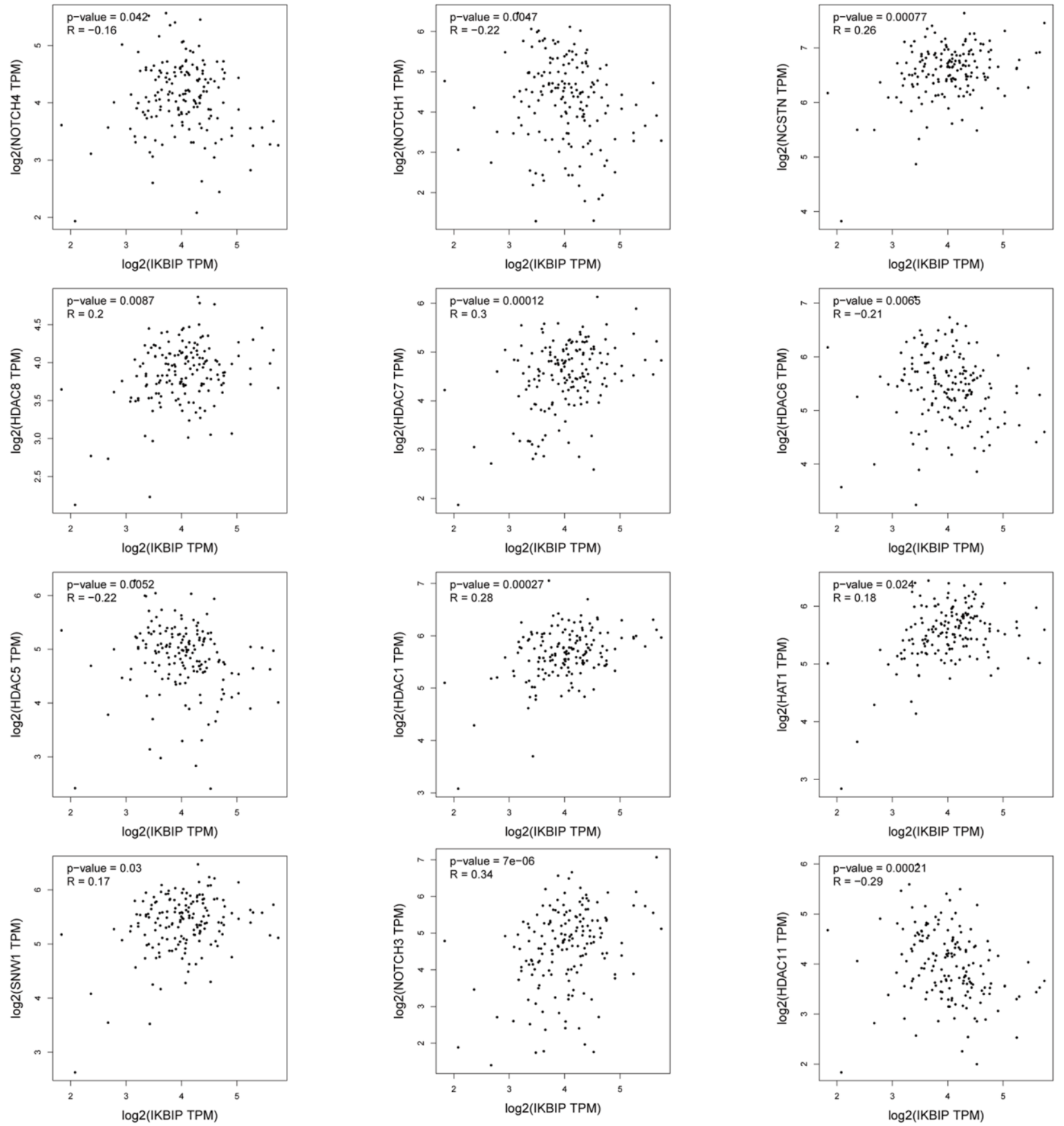

Supplementary Figure 11. Scatter plot shown genes in Notch signaling pathway related to IKBIP expression in glioma. 


\section{PI3K/Akt signaling pathway}
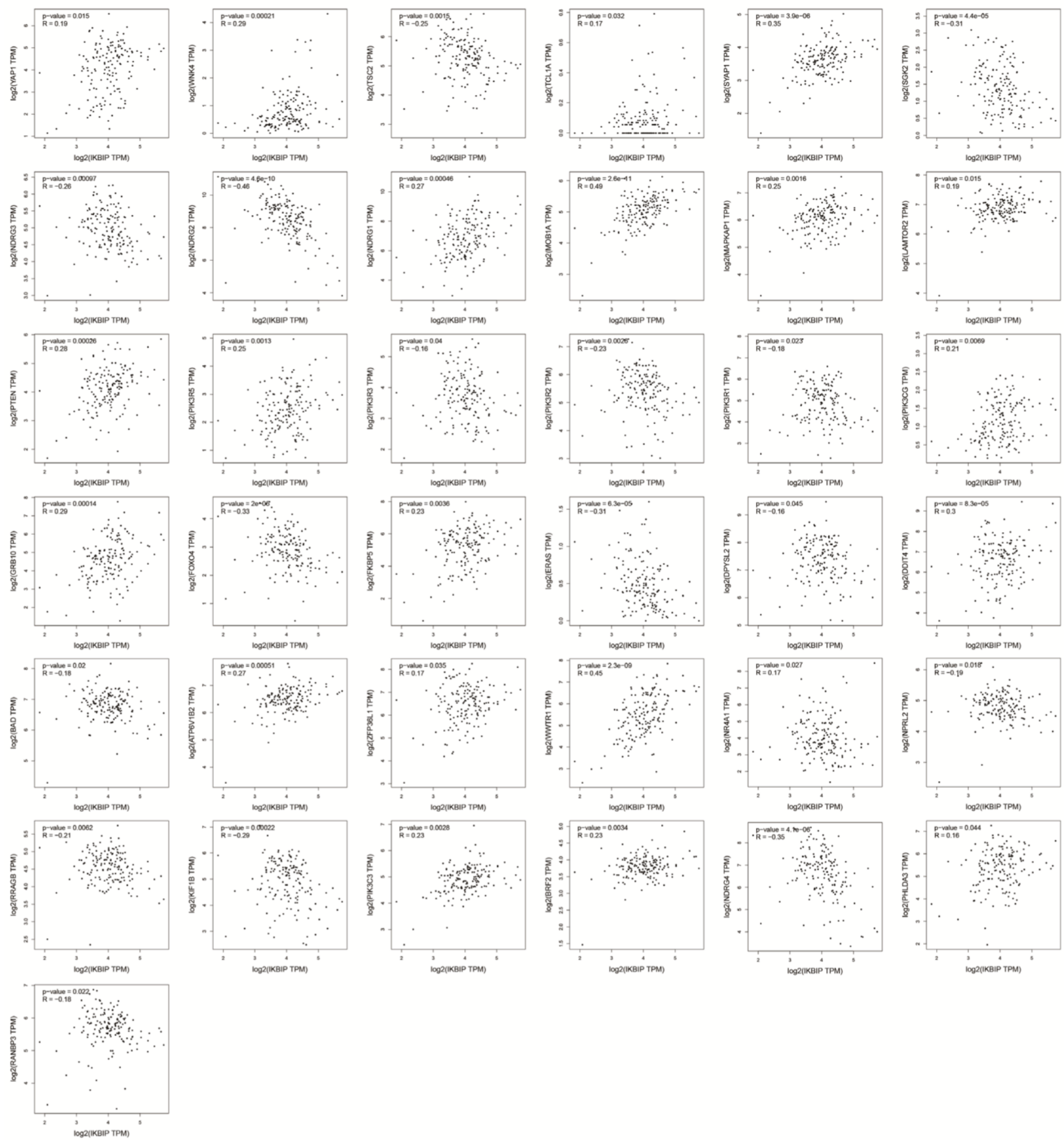

Supplementary Figure 12. Scatter plot shown genes in PI3K/Akt signaling pathway related to IKBIP expression in glioma. 


\section{TGF $\beta / S M A D$ signaling pathway}
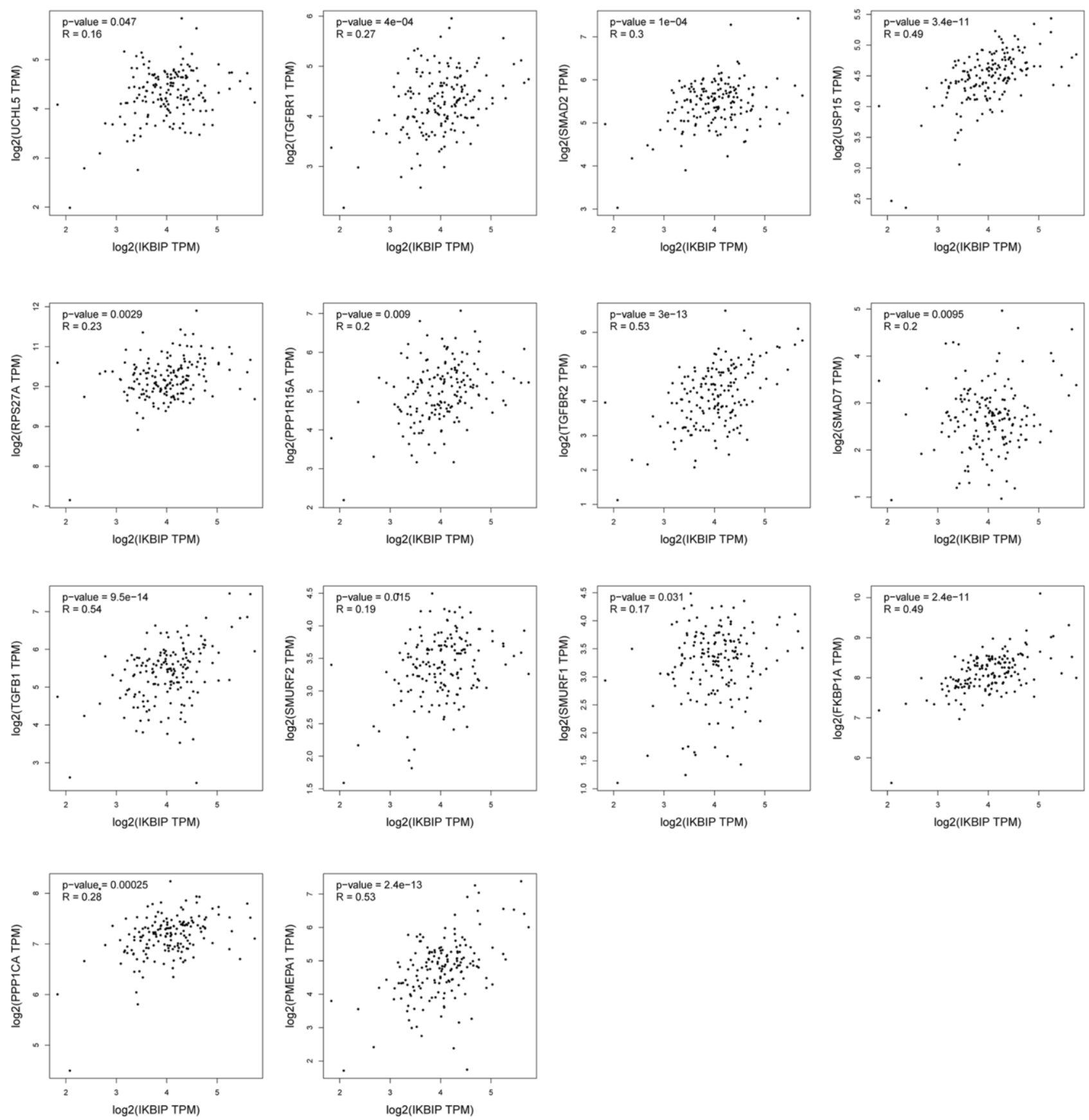

Supplementary Figure 13. Scatter plot shown genes in TGF $\beta /$ SMAD signaling pathway related to IKBIP expression in glioma. 


\section{Wnt/ß-catenin signaling pathway}
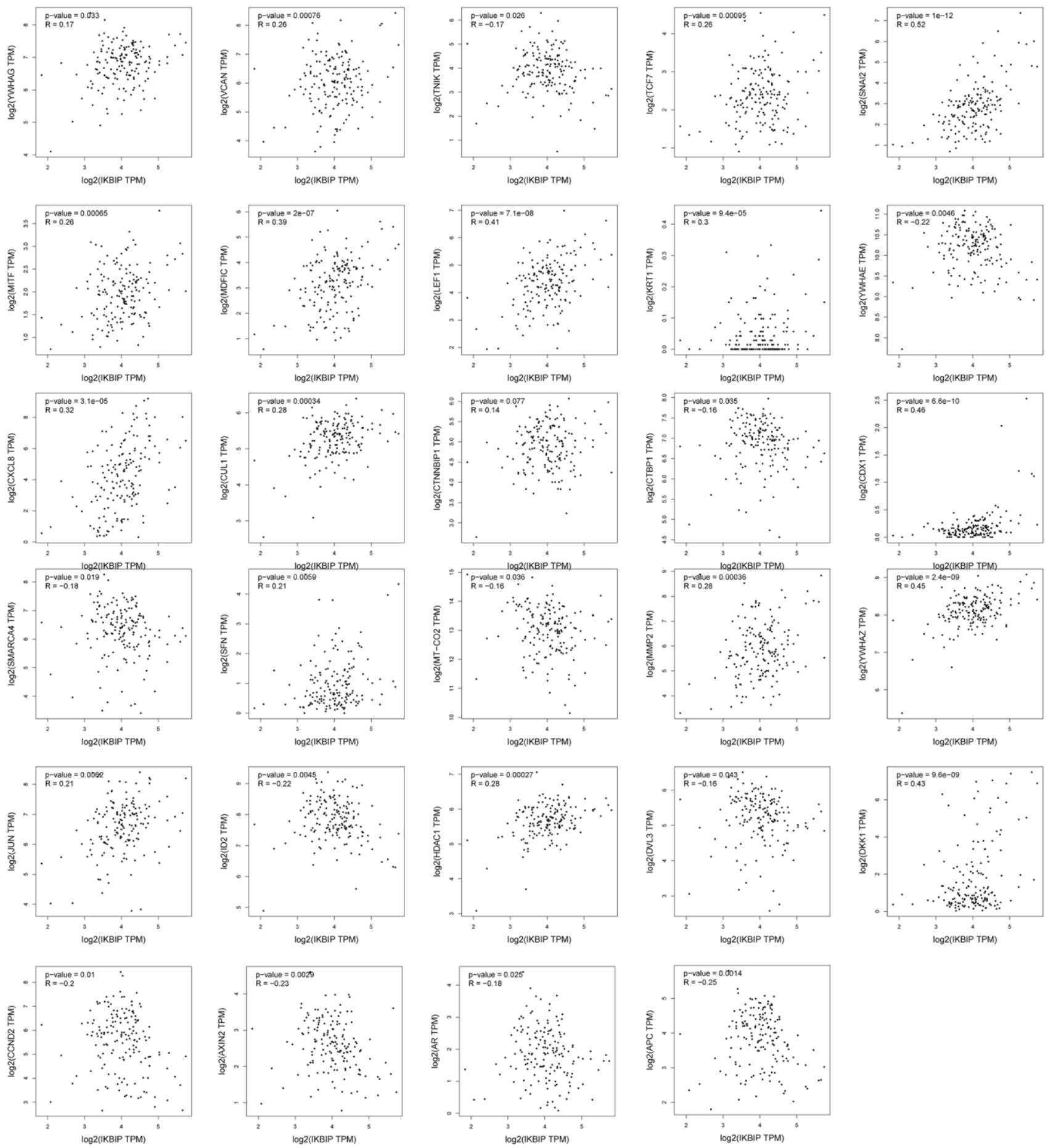

Supplementary Figure 14. Scatter plot shown genes in Wnt/ $\beta$-catenin signaling pathway related to IKBIP expression in glioma. 
signaling pathways

A

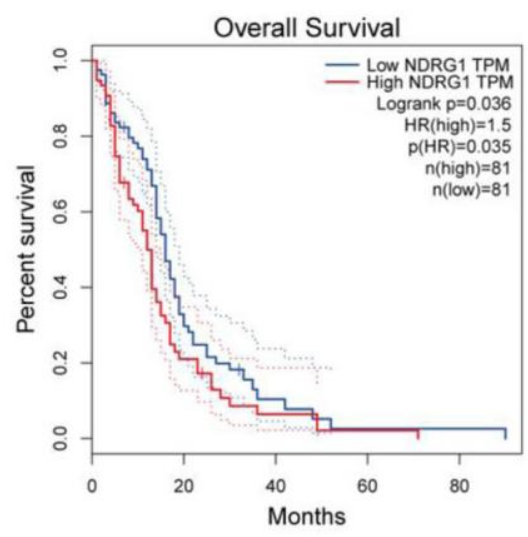

D
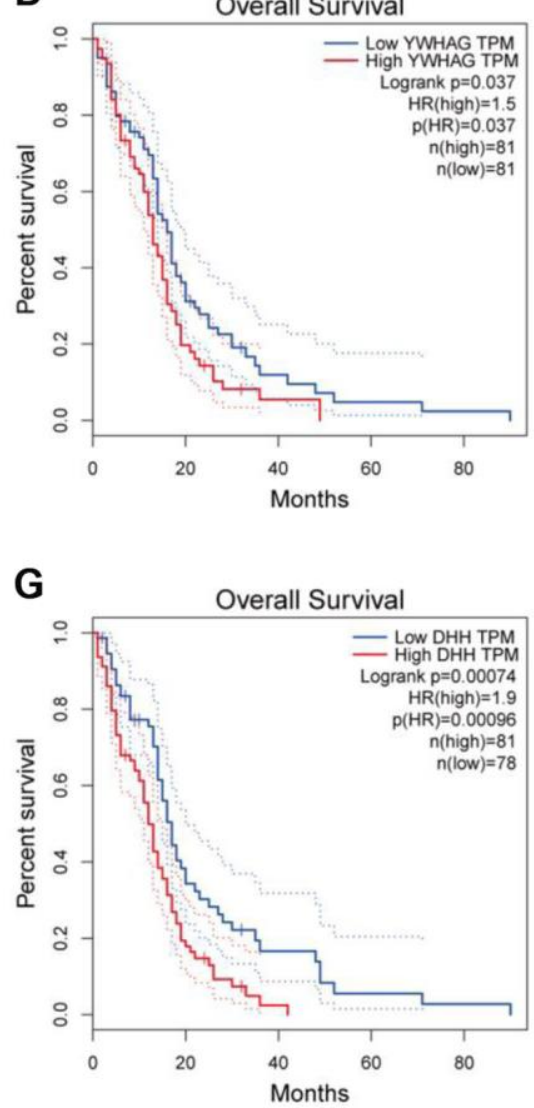

B

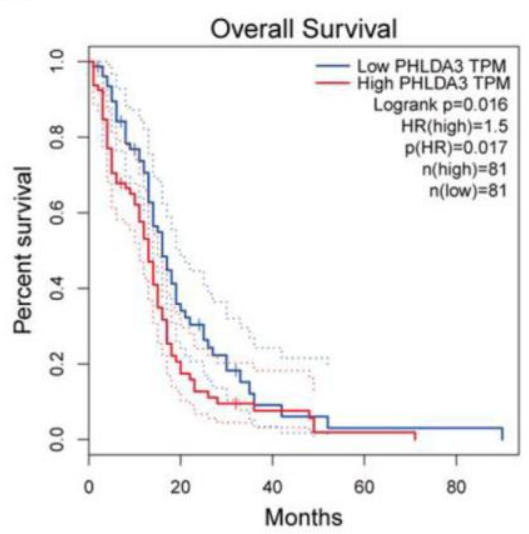

E

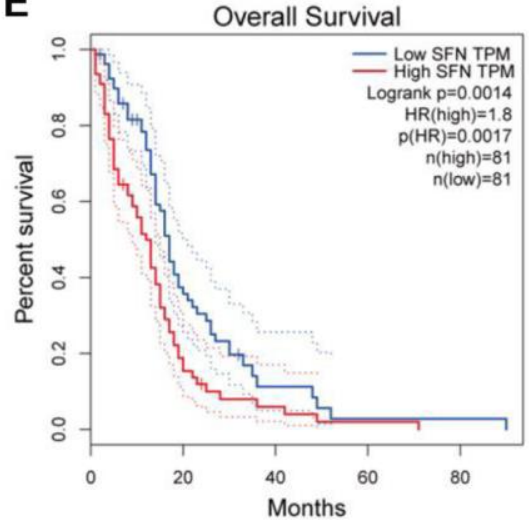

C

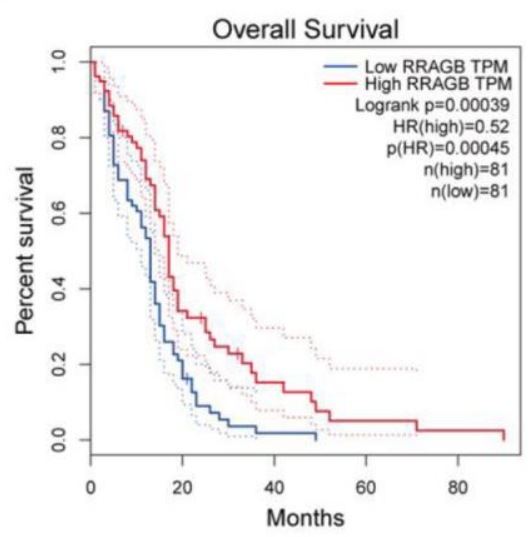

F

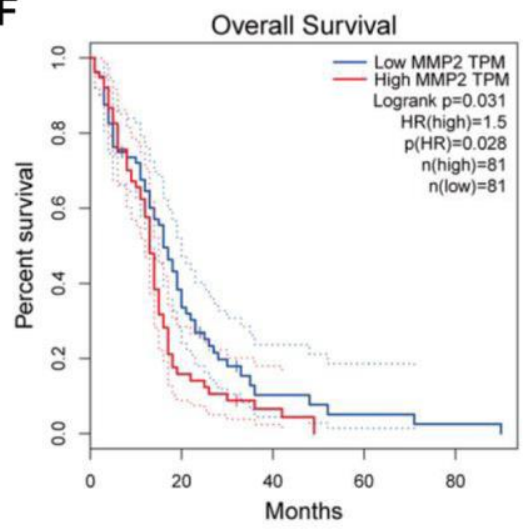

Supplementary Figure 15. Survival analysis shown the effect of genes related to IKBIP in the (A-C) PI3K/Akt signaling pathway, (D-F) Wnt/ $\beta$-catenin signaling pathway, and (G) Hedgehog signaling pathway to glioma patients. 